\title{
A Quasi-Variational Inequality Problem in Superconductivity
}

\author{
John W. Barrett \\ Department of Mathematics, Imperial College London \\ London $S W^{\prime}$ 2AZ, UK. \\ jwb@ic.ac.uk \\ Leonid Prigozhin \\ Department of Solar Energy and Environmental Physics, \\ Blaustein Institutes for Desert Research \\ Ben-Gurion University of the Negev, Sede Boqer Campus, 84990 Israel. \\ leonid@cs.bgu.ac.il \\ Received \\ Revised
}

\begin{abstract}
We derive a class of analytical solutions and a dual formulation of a scalar two space dimensional quasi-variational inequality problem in applied superconductivity. We approximate this formulation by a fully practical finite element method based on the lowest order Raviart-Thomas element, which yields approximations to both the primal and dual variables (the magnetic and electric fields). We prove subsequence convergence of this approximation, and hence prove existence of a solution to both the dual and primal formulations, for strictly star-shaped domains. The effectiveness of the approximation is illustrated by numerical examples with and without this domain restriction.

Keywords: Superconductivity; Kim model; quasi-variational inequalities; critical-state problems; mixed methods; finite elements, existence; convergence analysis.
\end{abstract}

AMS Subject Classification: 35D05, 35K85, 49J40, 49M29, 65M12, 65M60, 82C27

\section{Introduction}

\subsection{Primal problem}

Macroscopically, magnetisation of type-II superconductors can be regarded as an eddy current problem described by the Faraday and Ampère laws

$$
\partial_{t} \underline{b}+\underline{\nabla} \times \underline{e}=\underline{0}, \quad \underline{\nabla} \times \underline{b}=\underline{j},
$$

with a nonlinear and, often, multi-valued current-voltage relation characterizing the superconducting material. The magnetic permeability of superconductors is assumed equal to that of a vacuum and scaled to unity; hence, we will not distinguish between the magnetic field and the magnetic flux density $\underline{b}$. Typically, the currentvoltage relation represents the electric field inside the superconductor, $\underline{e}$, as the 
subgradient of a convex functional,

$$
\underline{e} \in \partial \Phi(\underline{j})
$$

where $j$ is the current density, see Bossavit ${ }^{9}$. To model the hysteretic response of superconductors to variations of the external magnetic fields and transport currents, it is convenient to formulate these problems as evolutionary variational or quasivariational inequalities, see Bossavit ${ }^{9}$ and Prigozhin ${ }^{22}$.

Of much interest, for technological applications and in physical experiments, are the energy loss estimates. Hence, the simultaneous determination of the current density and the electric field in a superconductor is often necessary. Unfortunately, the variational inequality formulations ${ }^{9,22}$, which we will call "primal", allow one to compute only the current density; and determining the electric field remains difficult, e.g. if the relation (1.2) is multi-valued. It has been shown recently, see Barrett and Prigozhin ${ }^{6}$, that a dual variational inequality formulation, based on an equivalent representation of the current-voltage relation,

$$
\underline{j} \in \partial \Phi^{*}(\underline{e})
$$

where $\Phi^{*}$ is the convex conjugate of $\Phi$, can be the basis for an efficient method for determining both of the variables in the Bean critical-state model ${ }^{8}$, which is the basic model for the magnetisation of type-II superconductors. Bean's model postulates that (in an isotropic superconductor) the current density cannot exceed some critical value, $|\underline{j}(\underline{x}, t)| \leq J_{c}$, the electric field is parallel to the current density, and is zero wherever $|\underline{j}(\underline{x}, t)|<J_{c}$. In this case $\Phi$ is the characteristic function of the set of admissible currents $\underline{K}_{0}$; that is,

$$
\Phi(\underline{v})=\chi_{\underline{K}_{0}}(\underline{v}):=\left\{\begin{array}{cll}
0 & \text { if } & \underline{v} \in \underline{K}_{0}, \\
+\infty & \text { if } & \underline{v} \notin \underline{K}_{0}
\end{array} .\right.
$$

In this paper we will study a similar dual formulation for the model in which the critical current density, $J_{c}$, depends on the magnetic field and the inequality becomes quasi-variational, see Prigozhin ${ }^{22}$. Such a modification of the Bean model, where $J_{c}=J_{c}(|\underline{b}|)$ is a monotonically decreasing function of the magnetic field, has been proposed by Kim et al. ${ }^{21}$ to account for the decrease of the magnetic moments in strong external fields, which is typical of most type-II superconductors. There are also materials demonstrating a secondary peak in their magnetisation hysteresis loops. The latter phenomenon, often called "the fishtail effect", can be described by the eddy current model with a non-monotonic $J_{c}(|\underline{b}|)$ dependance; see, e.g., Johansen et al. ${ }^{19}$. We mention here also the primal variational formulation for a generalized double critical-state model, see Badía and Lopez $^{2}$ and Kashima ${ }^{20}$, in which $\Phi$ is the characteristic function of the set of admissible currents satisfying $\underline{j}(\underline{x}, t) \in \underline{\Delta}(\underline{b}(\underline{x}, t))$ and $\underline{\Delta}(\underline{b}) \subset \mathbb{R}^{3}$ being a given family of closed convex sets.

Below we consider a simple geometric configuration of an infinite superconducting cylinder having a cross section $\Omega \subset \mathbb{R}^{2}$ and placed into a parallel non-stationary uniform external magnetic field $b_{e}(t)$. In this case the variational inequality for 
Bean's model, and the quasi-variational inequality for Kim's model, are most easily written in terms of the magnetic field which has only one non-zero component and can be regarded as a scalar function.

Let $\Omega$ be a bounded connected domain with a Lipschitz boundary $\partial \Omega$; if $\Omega$ is not simply connected we allow it to have a finite number of "holes" $\Omega_{i}, i=1 \rightarrow I$, and set $\Omega^{*}=\Omega \bigcup\left(\bigcup_{i=1 \rightarrow I} \overline{\Omega_{i}}\right)$. In this geometry, the induced magnetic field $w(\underline{x}, t)=$ $b(\underline{x}, t)-b_{e}(t)$ is zero on $\partial \Omega^{*}$, the outer boundary of $\Omega$, and depends only on time in each of the holes. We adopt the standard notation for curls in two dimensions: $\nabla \times \underline{v}(\underline{x})=\partial_{x_{1}} v_{2}(\underline{x})-\partial_{x_{2}} v_{1}(\underline{x})$ and $\underline{\nabla} \times v(\underline{x})=\left[\partial_{x_{2}} v(\underline{x}),-\partial_{x_{1}} v(\underline{x})\right]^{T}$.

To allow for at least some kind of spatial inhomogeneity on $\Omega$ we shall assume throughout the majority of this paper that

$$
J_{c}(\underline{x}, b)=k(\underline{x}) M(b),
$$

where $k \in C(\bar{\Omega})$, with $k(\underline{x}) \geq k_{0}>0$ for all $\underline{x} \in \bar{\Omega}$, and $M: \mathbb{R} \rightarrow\left[M_{0}, M_{1}\right] \subset \mathbb{R}$, with $M_{0}>0$, are given functions. We do not assume that $J_{c}$ is monotonically decreasing with respect to $|b|$, so we can deal with "the fishtail effect" mentioned above. In addition, we do not require $M$ to be continuous for our results on the dual formulation $(\mathrm{Q}),(1.15 \mathrm{a}, \mathrm{b})$; whereas we do require $M$ to be continuous for our results on the primal formulation $(\mathrm{P}),(1.8)$.

Using the laws (1.1) and the constitutive relation (1.2), we obtain for any $\eta \in$ $H_{0}^{1}\left(\Omega^{*}\right)$ that

$$
\begin{aligned}
\left(\partial_{t} b, \eta-w\right)_{\Omega^{*}} & =-(\nabla \times \underline{e}, \eta-w)_{\Omega^{*}}=-(\underline{e}, \underline{\nabla} \times(\eta-w))_{\Omega^{*}} \\
& \geq \Phi(\underline{\nabla} \times w)-\Phi(\underline{\nabla} \times \eta),
\end{aligned}
$$

where $(\cdot, \cdot)_{\Omega^{*}}$ is the standard inner product on $L^{2}\left(\Omega^{*}\right)$. In the Kim and similar models $\Phi=\chi_{\underline{K}_{0}(b)}$, the characteristic function of the set of admissible current densities

$$
\underline{K}_{0}(b):=\left\{\underline{\underline{v} \in\left[L^{2}\left(\Omega^{*}\right)\right]^{2}:} \begin{array}{c}
|\underline{v}(\underline{x})| \leq J_{c}(\underline{x}, b) \text { for a.e. } \underline{x} \in \Omega, \\
\underline{v}=\underline{0} \text { a.e. in } \Omega_{i}, i=1 \rightarrow I
\end{array}\right\} .
$$

Since, by Ampère's law, $\underline{j}=\underline{\nabla} \times b=\left[\partial_{x_{2}} b,-\partial_{x_{1}} b\right]^{T}$ we have that $|\underline{j}|=|\underline{\nabla} b|=|\underline{\nabla} w|$ and so one can replace the set of admissible currents in the variational formulation by the set of admissible induced magnetic fields. Hence, for any $b \in H^{1}\left(\Omega^{*}\right)$, we define

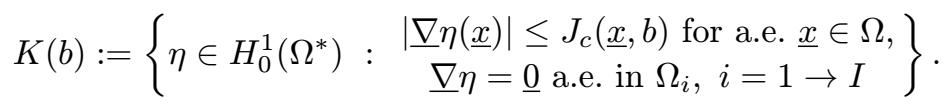

We therefore arrive at a primal variational formulation (quasi-variational inequality):

(P) Find $w \in L^{2}\left(0, T ; K\left(w+b_{e}\right)\right) \bigcap H^{1}\left(0, T ; L^{2}\left(\Omega^{*}\right)\right)$ such that $w(\cdot, 0)=w^{0}(\cdot)$ and

$$
\int_{0}^{T}\left(\partial_{t}\left(w+b_{e}\right), \eta-w\right)_{\Omega^{*}} \mathrm{~d} t \geq 0 \quad \forall \eta \in L^{2}\left(0, T ; K\left(w+b_{e}\right)\right) .
$$




\subsection{Analytical solution of primal problem}

For the special case of Bean's model, $M(\cdot) \equiv M_{0}>0$ in (1.5), the above inequality is variational, and its analytical solution for simply connected cross sections is known in the spatially homogeneous case $k(\cdot)=k_{0}>0$, see Barrett and Prigozhin ${ }^{5}$. We will now generalize this solution to the quasi-variational case under the additional assumption that, initially, the magnetic field depends only on the distance to the boundary of the domain $\Omega$, so that $w^{0}(\underline{x})=\mathcal{W}^{0}(\operatorname{dist}(\underline{x}, \partial \Omega))$; and then extend it, under a similar condition, to multiply connected domains $\Omega$. In applications, for the geometric configuration considered here, the initial magnetic field is usually uniform, so this condition is trivially satisfied.

Let $\Omega^{*} \equiv \Omega, J_{c}$ be given by (1.5) with $k \equiv 1$ and $M \in C\left(\mathbb{R},\left[M_{0}, M_{1}\right]\right)$, and $w^{0} \in$ $K\left(b^{0}\right)$, where $b^{0}(\underline{x})=w^{0}(\underline{x})+b_{e}(0)$. The latter condition implies that $\mathcal{W}^{0}(0)=0$ and $\left|d_{s} \mathcal{W}^{0}(s)\right| \leq M\left(\mathcal{B}^{0}(s)\right)$, where $\mathcal{B}^{0}(s)=\mathcal{W}^{0}(s)+b_{e}(0)$. Let us suppose first that $d_{t} b_{e}(t) \geq 0$ for all $t \geq 0$. Then we define $u(s, t)$ as a solution to the initial value problem

$$
\partial_{s} u=-M(u), \quad u(0, t)=b_{e}(t) .
$$

This yields that

$$
u(s, t)=F^{-1}\left(F\left(b_{e}(t)\right)-s\right),
$$

where $F(s)$ is such that $F^{\prime}(s)=[M(s)]^{-1}$ and $F(0)=0$. For all $t \geq 0$, on noting that $u(0, t)=b_{e}(t) \geq b_{e}(0)=\mathcal{B}^{0}(0)$, we define the nonnegative penetration depth

$$
d(t):=\sup \left\{s: u(r, t) \geq \mathcal{B}^{0}(r) \quad \forall r \in[0, s]\right\} .
$$

We then define

$$
\widetilde{b}(\underline{x}, t)=\mathcal{B}(\operatorname{dist}(\underline{x}, \partial \Omega), t), \quad \text { where } \quad \mathcal{B}(s, t):= \begin{cases}u(s, t) & \text { if } s \in[0, d(t)], \\ \mathcal{B}^{0}(s) & \text { if } s>d(t) .\end{cases}
$$

We claim that $\widetilde{w}(\underline{x}, t)=\widetilde{b}(\underline{x}, t)-b_{e}(t)$ solves the quasi-variational inequality $(\mathrm{P})$.

Obviously, $\left.\widetilde{w}\right|_{\partial \Omega}=0$. If $d(0)=0$ then $\mathcal{B}(s, 0) \equiv \mathcal{B}^{0}(s)$ and $\widetilde{w}(\underline{x}, 0)=w^{0}(\underline{x})$. In addition, since $d_{s} \mathcal{B}^{0}(s) \geq-M\left(\mathcal{B}^{0}(s)\right)$ and $\mathcal{B}^{0}(0)=b_{e}(0)$, we obtain that $F\left(\mathcal{B}^{0}(s)\right) \geq$ $F\left(b_{e}(0)\right)-s=F(u(s, 0))$. Due to the monotonicity of $F$, this yields that $\mathcal{B}^{0}(s) \geq$ $u(s, 0)$. Hence, if $d(0)>0$ the equality $u(s, 0)=\mathcal{B}^{0}(s)$ holds for $0 \leq s \leq d(0)$ and so once again $\mathcal{B}(s, 0) \equiv \mathcal{B}^{0}(s)$ and $\widetilde{w}(\underline{x}, 0)=w^{0}(\underline{x})$. Next, on setting $\Omega_{T}:=\Omega \times(0, T)$, we define

$$
\Omega_{T}^{+}:=\left\{(\underline{x}, t) \in \Omega_{T}: \operatorname{dist}(\underline{x}, \partial \Omega) \leq d(t)\right\} .
$$

Then we have a.e. on $\Omega_{T}^{+}$that $\underline{\nabla} \widetilde{w}(\underline{x}, t)=\left.\partial_{s} u(s, t)\right|_{s=\operatorname{dist}(\underline{x}, \partial \Omega)} \underline{\nabla}[\operatorname{dist}(\underline{x}, \partial \Omega)]$, and so $|\underline{\nabla} \widetilde{w}(\underline{x}, t)|=\left|\partial_{s} u(s, t)\right|_{s=\operatorname{dist}(\underline{x}, \partial \Omega)}=M(\widetilde{b}(\underline{x}, t))$. Whereas on $\Omega_{T}^{-}:=\Omega_{T} \backslash \Omega_{T}^{+}$, we have that $\widetilde{w}(\underline{x}, t)=\mathcal{W}^{0}(\operatorname{dist}(\underline{x}, \partial \Omega))+b_{e}(0)-b_{e}(t)$, and so it is easily deduced that $|\underline{\nabla} \widetilde{w}(\underline{x}, t)| \leq M(\widetilde{b}(\underline{x}, t))$. Therefore $\widetilde{w} \in K(\widetilde{b})$. As $d_{t} b_{e}(t) \geq 0$ for all $t \geq 0$, it follows from (1.9) and (1.10) that $\partial_{t} u(s, t) \geq 0, d_{t} d(t) \geq 0$, and so $\partial_{t} \widetilde{b} \geq 0$ a.e. on $\Omega_{T}$. Furthermore, since $\partial_{t} \widetilde{b}=0$ on $\Omega_{T}^{-}$, to prove the inequality (1.8) we need to show 
that $\int_{\Omega_{T}^{+}} \partial_{t} \widetilde{b}(\eta-\widetilde{w}) \mathrm{d} \underline{x} \mathrm{~d} t \geq 0$ for all $\eta \in K(\widetilde{b})$. We note that functions from the convex set $K(\widetilde{b})$ vanish on $\partial \Omega$, and $\widetilde{w} \in K(\widetilde{b})$ decreases with distance from $\partial \Omega$ on $\Omega_{T}^{+}$at the maximal possible rate, $M(\widetilde{b})$, for a function in $K(\widetilde{b})$. Hence we conclude that $\eta-\widetilde{w} \geq 0$ in $\Omega_{T}^{+}$for any $\eta \in K(\widetilde{b})$, and so the inequality (1.8) is satisfied.

If $d_{t} b_{e}(t) \leq 0$ for all $t \geq 0$, an analytical solution can be found in a similar way. Now $u(s, t)$ is a solution to $\partial_{s} u=M(u)$ with $u(0, t)=b_{e}(t)$, and the penetration depth determined as $d(t):=\sup \left\{s: u(r, t) \leq \mathcal{B}^{0}(r) \quad \forall r \in[0, s]\right\}$. Finally, since at each moment in time these solutions depend only on the distance to the domain boundary, we can construct an analytical solution to (P) for arbitrary $b_{e} \in H^{1}(0, T)$ by combining the solutions described above.

Solution for a multiply connected domain can be constructed in a similar way by regarding the hole closures, $\overline{\Omega_{i}} \subset \Omega^{*}$, as free Dirichlet sets (see Buttazzo and Stepanov ${ }^{11}$ ) and, correspondingly, defining the distance from a point $\underline{x} \in \Omega^{*}$ to the domain boundary as

$$
\begin{aligned}
& \operatorname{dist}_{D}\left(\underline{x}, \partial \Omega^{*}\right) \\
& \quad:=\inf \left\{\int_{0}^{1} k(s(t))\left|d_{t} s(t)\right| \mathrm{d} t: s \in C^{0,1}\left([0,1] ; \Omega^{*}\right), s(0)=\underline{x}, s(1) \in \partial \Omega^{*}\right\},
\end{aligned}
$$

where

$$
k(\underline{x}):=\left\{\begin{array}{lll}
1 & \text { if } & \underline{x} \in \Omega \\
0 & \text { if } & \underline{x} \in \bigcup_{i=1}^{I} \overline{\Omega_{i}} .
\end{array}\right.
$$

Obviously, $\operatorname{dist}_{D}\left(\underline{x}, \partial \Omega^{*}\right)$ is the same for all points in each hole and, for $\underline{x} \in \Omega$, the distance is the length of the shortest path to the outer boundary of $\Omega$ assuming the parts of a path inside holes are not counted. Assuming, for a multiply connected cross section, that the initial magnetic field is a function of $\operatorname{dist}_{D}\left(\underline{x}, \partial \Omega^{*}\right)$ and taking into account that $\left|\underline{\nabla} \operatorname{dist}_{D}\left(\underline{x}, \partial \Omega^{*}\right)\right|=1$ a.e. in $\Omega$, one can now build an analytical solution to $(\mathrm{P})$ exactly as above but using the distance function (1.11).

\subsection{Dual problem}

The primal formulation allows one to calculate the magnetic field and, by Ampère's law, also the current density in a superconductor. Nevertheless, determining the electric field remains difficult and, to solve this problem for the Bean model, several approaches have been proposed ${ }^{10,3,6,14}$. Here we derive a dual (mixed) formulation for models with critical current density depending on the magnetic field.

Returning to possibly spatially inhomogeneous $J_{c}$ defined by (1.5), and recalling the definition of $F$ in (1.9), $F^{\prime}(s)=[M(s)]^{-1}$ and $F(0)=0$, we have that the condition $\underline{j}=\underline{\nabla} \times b \in \underline{K}_{0}(b)$ is equivalent to

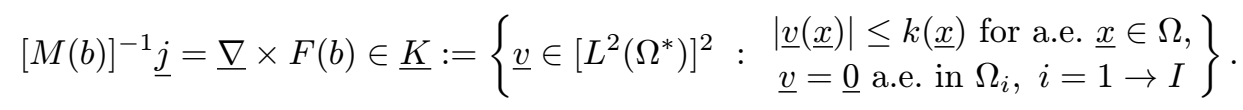


Note that $\underline{e} \in \partial \chi_{\underline{K}_{0}(b)}(\underline{j})$ means that $\underline{j} \in \underline{K}_{0}(b)$ and $(\underline{e}, \underline{i}-\underline{j})_{\Omega^{*}} \leq 0$ for any $\underline{i} \in \underline{K}_{0}(b)$. The latter is possible if and only if $\underline{e} \cdot(\underline{i}-\underline{j}) \leq 0$ a.e. in $\Omega^{*}$ for any $\underline{i} \in \underline{K}_{0}(b)$. Hence $\left(\underline{e}, \underline{v}-[M(b)]^{-1} \underline{j}\right)_{\Omega^{*}} \leq 0$ for any $\underline{v} \in \underline{K}$ and the current-voltage relation (1.2), for the choice of $\Phi=\chi_{\underline{K}_{0}(b)}$, can be rewritten as

$$
\begin{gathered}
\underline{e} \in \partial \chi_{\underline{K}}(\underline{\nabla} \times F(b)) \quad \text { with its dual form as } \quad \underline{\nabla} \times F(b) \in \partial \chi_{\underline{K}}^{*}(\underline{e}), \\
\text { where } \quad \chi_{\underline{K}}^{*}(\underline{e}):=\sup _{\underline{v} \in\left[L^{2}\left(\Omega^{*}\right)\right]^{2}}\left\{(\underline{e}, \underline{v})_{\Omega^{*}}-\chi_{\underline{K}}(\underline{v})\right\}=\int_{\Omega} k|\underline{e}| \mathrm{d} \underline{x} .
\end{gathered}
$$

The dual form (1.12) yields for any test field $\underline{u}$ that

$$
(\underline{\nabla} \times F(b), \underline{u}-\underline{e})_{\Omega^{*}} \leq \int_{\Omega} k(|\underline{u}|-|\underline{e}|) \mathrm{d} \underline{x} .
$$

We set $\underline{u}=\mathcal{R} \underline{v}$ and $\underline{e}=\mathcal{R} \underline{q}$, where $\mathcal{R}\left[p_{1}, p_{2}\right]^{T}:=\left[p_{2},-p_{1}\right]^{T}$. Since $(\underline{\nabla} \times$ $F(b), \mathcal{R}(\underline{v}-\underline{q}))_{\Omega^{*}}=(\underline{\nabla}[F(b)], \underline{v}-\underline{q})_{\Omega^{*}}$ and $b-b_{e}(t) \in H_{0}^{1}\left(\Omega^{*}\right)$, we obtain for all test fields $\underline{v}$ that

$$
\int_{\Omega} k(|\underline{v}|-|\underline{q}|) \mathrm{d} \underline{x}+\left(F(b)-F\left(b_{e}\right), \underline{\nabla} \cdot(\underline{v}-\underline{q})\right)_{\Omega^{*}} \geq 0 .
$$

Similarly, Faraday's law takes the form $\partial_{t} b-\underline{\nabla} \cdot \underline{q}=0$.

We introduce the Banach space

$$
\underline{V}^{\mathcal{M}}(D):=\left\{\underline{v} \in[\mathcal{M}(\bar{D})]^{2}: \underline{\nabla} \cdot \underline{v} \in L^{2}(D)\right\},
$$

where $\mathcal{M}(\bar{D})$ is the Banach space of bounded Radon measures; i.e. $\mathcal{M}(\bar{D}) \equiv$ $[C(\bar{D})]^{*}$, the dual of $C(\bar{D})$. Our mixed formulation of $(\mathrm{P}),(1.8)$, is then:

(Q) Find $\underline{q} \in L^{2}\left(0, T ; \underline{V}^{\mathcal{M}}\left(\Omega^{*}\right)\right)$ and $b \in H^{1}\left(0, T ; L^{2}\left(\Omega^{*}\right)\right)$ such that $b(\cdot, 0)=b^{0}(\cdot)$ and

$$
\begin{gathered}
\int_{0}^{T}\left(\partial_{t} b-\underline{\nabla} \cdot \underline{q}, \eta\right)_{\Omega^{*}} \mathrm{~d} t=0 \quad \forall \eta \in L^{2}\left(\Omega_{T}^{*}\right), \\
\int_{0}^{T}\left[\int_{\bar{\Omega}} k(|\underline{v}|-|\underline{q}|)+\left(F(b)-F\left(b_{e}\right), \underline{\nabla} \cdot(\underline{v}-\underline{q})\right)_{\Omega^{*}}\right] \mathrm{d} t \geq 0 \\
\forall \underline{v} \in L^{2}\left(0, T ; \underline{V}^{\mathcal{M}}\left(\Omega^{*}\right)\right) ;(1
\end{gathered}
$$

where $b^{0}(\cdot)=w^{0}(\cdot)+b_{e}(0)$ and $\Omega_{T}^{*}:=\Omega^{*} \times(0, T)$.

Clearly, if the pair $\{\underline{q}, b\}$ satisfies (Q), it follows from (1.15b) that $\underline{\nabla}(F(b)-$ $\left.F\left(b_{e}\right)\right)=0$ in each hole $\Omega^{i}$ and so $\underline{\nabla} b=0$ there as well. In addition, $\{\underline{q}+\underline{u}, b\}$ is also a solution of $(\mathrm{Q})$ if $\operatorname{supp}(\underline{u}) \subset \bigcup_{i=1 \rightarrow I} \Omega^{i}$ and $\underline{\nabla} \cdot \underline{u}=0$. This corresponds to the well-known fact that the eddy current problem does not determine the electric field in non-conducting media in a unique way. Furthermore, it follows immediately from $(1.15 \mathrm{a}, \mathrm{b})$ that a solution $\{q, b\}$ of $(\mathrm{Q})$ is such that

$\underline{q} \in \underline{K}_{1}\left(\partial_{t} b\right):=\left\{\underline{v} \in L^{2}\left(0, T ; \underline{V}^{\overline{\mathcal{M}}}\left(\Omega^{\star}\right)\right):(\underline{\nabla} \cdot \underline{v}, \eta)_{\Omega_{T}^{\star}}=\left(\partial_{t} b, \eta\right)_{\Omega_{T}^{\star}} \quad \forall \eta \in L^{2}\left(\Omega_{T}^{\star}\right)\right\}$ and

$$
\int_{0}^{T} \int_{\bar{\Omega}} k|\underline{q}|=\min _{\underline{v} \in \underline{K}_{1}\left(\partial_{t} b\right)} \int_{0}^{T} \int_{\bar{\Omega}} k|\underline{v}| .
$$


One can exploit this formulation to obtain $\underline{q}$ if $\partial_{t} b$ is known. As was shown above, if $k \equiv 1$ in $\Omega$ and the initial magnetic field is a function of $\operatorname{dist}_{D}\left(\underline{x}, \partial \Omega^{*}\right)$, an analytical solution is known for the solution $b$ of the primal formulation $(\mathrm{P})$; that is, $b(\underline{x}, t)=$ $\mathcal{B}\left(\operatorname{dist}_{D}\left(\underline{x}, \partial \Omega^{*}\right), t\right)$. One could use this $b$ in $(1.16)$ to recover $\underline{q}$. Problems, similar to (1.16), and their dual formulations have been considered, for simply connected domains, in several works ${ }^{24,17,18,12,14}$. We only mention here that the ridge (cut locus) of the domain plays an important role in the analysis in these works and that an integral representation of the solution to (1.16) with $k \equiv 1$ has been derived for $\Omega$ being a polygonal domain ${ }^{18}$ and for domains with a smooth boundary ${ }^{12,14}$.

In our analysis of problem (Q) presented below we will also restrict ourselves to the case when $\Omega^{*} \equiv \Omega$ and, moreover, assume that the domain $\Omega$ is strictly star-shaped. The later assumption is to ensure that certain density results hold; see $(1.21)$ and $(1.22 \mathrm{a}-\mathrm{c})$ below. However, our numerical method applies to the case of a multiply connected cross section and we will present a numerical example. In addition, to avoid perturbation of domain errors in our finite element approximation, we will assume that $\Omega$ is polygonal for ease of exposition.

The outline of this paper is as follows. In the next section we introduce a regularized version, $\left(\mathrm{Q}_{r}\right)$, of our mixed formulation, $(\mathrm{Q})$, by smoothing the nondifferentiable functional $\int_{\Omega_{T}}|\underline{v}|$ by replacing $|\underline{v}|$ with $\frac{1}{r}|\underline{v}|^{r}$ with $r>1$. We then consider the finite element approximation, $\left(\mathrm{Q}_{r}^{h}, \tau\right)$, of $\left(\mathrm{Q}_{r}\right)$ using Raviart-Thomas elements of the lowest order with vertex sampling on the nonlinear term. We then establish stability bounds on this approximation, independent of the mesh parameter $h$, time step parameter $\tau$ and the regularization parameter $r$. In Section 3, under the assumption (1.5), we prove subsequence convergence of this approximation, as the parameters, $h$ and $\tau$, go to zero and $r$ goes to one, and establish existence of a solution $\{\underline{q}, b\}$ to $(\mathrm{Q})$. Moreover, on further assuming that $M \in C\left(\mathbb{R},\left[M_{0}, M_{1}\right]\right)$, we show that $w=b-b_{e}$ is a solution of $(\mathrm{P})$. Finally in Section 4 we present some numerical experiments based on the discretization $\left(\mathrm{Q}_{r}^{h, \tau}\right)$.

This paper extends the dual formulation in Barrett and Prigozhin ${ }^{6}$ from variational to quasi-variational inequalities. We introduce, and prove the convergence of, a fully practical numerical scheme based on the dual formulation, (Q), which enables one to approximate simultaneously both the electric and magnetic fields. Hence, approximating the local rate of energy dissipation, $\underline{j} \cdot \underline{e}=J_{c}(\underline{x}, b)|\underline{e}|$, becomes straightforward. In proving existence of a solution to the dual formulation (Q), we also show existence of a solution to the primal quasi-variational inequality (P) involving a gradient constraint. We are not aware of any previous existence results for $(\mathrm{P})$ in the literature, apart from the analytical solution above which holds for $k \equiv 1$ and special initial data $w^{0}$. However, existence results are available in the case $k \equiv 1$ and $M \in C\left(\mathbb{R},\left[M_{0}, M_{1}\right]\right)$ for a modified problem $\left(\mathrm{P}_{p}\right)$ which includes a $p$-Laplacian term, and this term plays a crucial role in the analysis ${ }^{23,1}$. Finally, we note that there are existence results ${ }^{20}$ for a primal quasi-variational $3 \mathrm{~d}$ formulation of the double critical-state model where two different constants, $J_{c \|}$ and $J_{c \perp}$, limit 
the magnitudes, respectively, of the parallel and orthogonal (to the magnetic field) components of the current density.

\subsection{Notation}

We end this section with a few remarks about the notation employed in this paper. Above and throughout we adopt the standard notation for Sobolev spaces on a bounded domain $D$ with a Lipschitz boundary, denoting the norm of $W^{\ell, p}(D)(\ell \in$ $\mathbb{N}, p \in[1, \infty])$ by $\|\cdot\|_{\ell, p, D}$ and the semi-norm by $|\cdot|_{\ell, p, D}$. Of course, we have that $|\cdot|_{0, p, D} \equiv\|\cdot\|_{0, p, D}$. We extend these norms and semi-norms in the natural way to the corresponding spaces of vector functions. For $p=2, W^{\ell, 2}(D)$ will be denoted by $H^{\ell}(D)$ with the associated norm and semi-norm written as, respectively, $\|\cdot\|_{\ell, D}$ and $|\cdot|_{\ell, D}$. We set $W_{0}^{1, p}(D):=\left\{\eta \in W^{1, p}(D): \eta=0\right.$ on $\left.\partial D\right\}$, and $H_{0}^{1}(D) \equiv W_{0}^{1,2}(D)$. $|D|$ will denote the measure of $D$ and $(\cdot, \cdot)_{D}$ the standard inner product on $L^{2}(D)$. When $D \equiv \Omega$, for ease of notation we write $(\cdot, \cdot)$ for $(\cdot, \cdot)_{\Omega}$.

Let $C(\bar{D})$ denote the space of continuous functions on $\bar{D}$. As one can identify $L^{1}(D)$ as a closed subspace of the Banach space of bounded Radon measures, $\mathcal{M}(\bar{D}) \equiv[C(\bar{D})]^{\star} ;$ it is convenient to adopt the notation

$$
\int_{\bar{D}}|\mu| \equiv\|\mu\|_{\mathcal{M}(\bar{D})}:=\sup _{\substack{\eta \in C(\bar{D}) \\|\eta|_{0, \infty, D} \leq 1}}\langle\mu, \eta\rangle_{C(\bar{D})}<\infty
$$

where $\langle\cdot, \cdot\rangle_{C(\bar{D})}$ denotes the duality pairing on $[C(\bar{D})]^{\star} \times C(\bar{D})$. The condition $\underline{\nabla} . \underline{v} \in L^{2}(D)$ in $(1.14)$ means that there exists $u \in L^{2}(D)$ such that $\langle\underline{v}, \underline{\nabla} \phi\rangle_{C(\bar{D})}=$ $-(u, \phi)_{D}$ for any $\phi \in C_{0}^{1}(D)$.

We note that if $\left\{\mu_{n}\right\}_{n \geq 0}$ is a bounded sequence in $\mathcal{M}(\bar{D})$, then there exist a subsequence $\left\{\mu_{n_{j}}\right\}_{n_{j} \geq 0}$ and a $\mu \in \mathcal{M}(\bar{D})$ such that as $n_{j} \rightarrow \infty$

$$
\mu_{n_{j}} \rightarrow \mu \quad \text { vaguely in } \mathcal{M}(\bar{D}) ; \quad \text { i.e. } \quad\left\langle\mu_{n_{j}}-\mu, \eta\right\rangle_{C(\bar{D})} \rightarrow 0 \quad \forall \eta \in C(\bar{D}) .
$$

Moreover, we have that

$$
\liminf _{n_{j} \rightarrow \infty} \int_{\bar{D}}\left|\mu_{n_{j}}\right| \geq \int_{\bar{D}}|\mu|
$$

see e.g. page 223 in Folland ${ }^{16}$.

As well as the Banach space $\underline{V}^{\mathcal{M}}(D)$, recall (1.14), we require for a given $s \in$ $[1, \infty]$ the Banach space

$$
\underline{V^{s}}(D):=\left\{\underline{v} \in\left[L^{s}(D)\right]^{2}: \underline{\nabla} \cdot \underline{v} \in L^{2}(D)\right\} .
$$

We note that $\underline{V}^{\mathcal{M}}(D)$ and $\underline{V}^{s}(D)$ for $s \in[1,2)$ are not of local type; that is, $\underline{v} \in \underline{V}^{\mathcal{M}}(D)\left[\underline{V}^{s}(D)\right]$ and $\phi \in C^{\infty}(\bar{D})$ does not imply that $\phi \underline{v} \in \underline{V}^{\mathcal{M}}(D)\left[\underline{V}^{s}(D)\right]$, see e.g. page 22 in Temam ${ }^{25}$. Therefore, one has to avoid cut-off functions in proving any required density results. If $\Omega$ is strictly star-shaped, which we shall assume for 
the analysis in this paper, one can show, using the standard techniques of change of variable and mollification, that

$$
\left[C^{\infty}(\bar{\Omega})\right]^{2} \quad \text { is dense in } \quad \underline{V}^{s}(\Omega) \quad \text { if } \quad s \in(1, \infty) .
$$

Moreover, for any $\underline{v} \in \underline{V}^{\mathcal{M}}(\Omega)$, there exist $\left\{\underline{v}_{j}\right\}_{j \geq 1} \in\left[C^{\infty}(\bar{\Omega})\right]^{2}$ such that

$$
\begin{aligned}
& \underline{v}_{j} \rightarrow \underline{v} \quad \text { vaguely in }[\mathcal{M}(\bar{\Omega})]^{2} \quad \text { as } j \rightarrow \infty, \\
& \underline{\nabla} \cdot \underline{v}_{j} \rightarrow \underline{\nabla} \cdot \underline{v} \quad \text { weakly in } L^{2}(\Omega) \quad \text { as } j \rightarrow \infty \text {, } \\
& \limsup _{j \rightarrow \infty} \int_{\Omega} \rho\left|\underline{v}_{j}\right| \mathrm{d} \underline{x}=\int_{\bar{\Omega}} \rho|\underline{v}|
\end{aligned}
$$

for any positive $\rho \in C(\bar{\Omega})$. We briefly outline the proofs of (1.21) and (1.22a-c). Without loss of generality, one can assume that $\Omega$ is strictly star-shaped with respect to the origin. Then for $\underline{v}$ defined on $\Omega$ and $\theta>1$, we have that $\underline{v}_{\theta}(\underline{x})=\underline{v}\left(\theta^{-1} \underline{x}\right)$ is defined on $\Omega_{\theta}:=\theta \Omega \supset \Omega$. Applying standard Friedrich's mollifiers $J_{\varepsilon}$ to $\underline{v}_{\theta}$, and a diagonal subsequence argument yield, for $\theta \rightarrow 1$ and $\varepsilon \rightarrow 0$ as $j \rightarrow \infty$, the desired sequences $\{\underline{v}\}_{j \geq 1}$ demonstrating (1.21) if $\underline{v} \in \underline{V}^{s}(\Omega)$ and satisfying (1.22a-c) if $\underline{V}^{\mathcal{M}}(\Omega)$; see e.g. Lemma 2.4 in Barrett and Prigozhin ${ }^{7}$, where such techniques are used to prove similar density results.

Finally, throughout $C$ denotes a generic positive constant independent of the regularization parameter, $r \in(1, \infty)$, the mesh parameter $h$ and the time step parameter $\tau$. Whereas, $C(s)$ denotes a positive dependent on the parameter $s$.

\section{Numerical Approximation of (Q)}

First, we gather together our assumptions on the data.

(A1) Let $\Omega \subset \mathbb{R}^{2}$ be a strictly star-shaped domain with boundary $\partial \Omega$, so that $\Omega^{*} \equiv \Omega$ in $(\mathrm{P}),(1.8)$, and (Q), (1.15a,b). Let $b_{e} \in H^{1}(0, T), k \in C(\bar{\Omega})$ with $k_{1} \geq$ $k(\underline{x}) \geq k_{0}>0$ for all $\underline{x} \in \bar{\Omega}$, and $M: \mathbb{R} \rightarrow\left[M_{0}, M_{1}\right] \subset \mathbb{R}$ with $M_{0}>0$. We shall assume that $b^{0}(\cdot)=w^{0}(\cdot)+b_{e}(0)$ with $w^{0} \in K\left(b^{0}\right)$; i.e. $b^{0}(\cdot)-b_{e}(0) \in H_{0}^{1}(\Omega)$ and $\left|\underline{\nabla} b^{0}\right| \leq k M\left(b^{0}\right)$ a.e. in $\Omega$.

We note that the assumptions (A1) do allow for an $M(s)$ that is strictly positive on any bounded interval of $\mathbb{R}$, but goes to zero as $|s| \rightarrow \infty$. This follows since a solution of $(\mathrm{P})$ is such that $w=b-b_{e}=0$ on $\partial \Omega \times(0, T)$ and $|\underline{\nabla} w| \leq k_{1} M_{1}$ a.e. in $\Omega_{T}$. Hence it follows that $|b| \leq \max _{t \in[0, T]}\left|b_{e}(t)\right|+k_{1} M_{1} \operatorname{diam}(\Omega)$ a.e. on $\Omega_{T}$. Therefore an $M(s)$ that is strictly positive on any bounded interval, but goes to zero as $|s| \rightarrow \infty$ can always be replaced by

$$
M_{L}(s):= \begin{cases}M(s) & \text { if }|s| \leq L, \\ M(L)>0 & \text { if }|s| \geq L \geq k_{1} M_{1} \operatorname{diam}(\Omega)+\max _{t \in[0, T]}\left|b_{e}(t)\right|\end{cases}
$$

without changing the problem $(\mathrm{P})$.

In order to prove existence of, and approximate, solutions to (Q), (1.15a,b), we 
introduce $F \in C(\mathbb{R}), G \in C^{1}(\mathbb{R})$ such that

$$
F(0)=G(0)=0, \quad F^{\prime}(s)=[M(s)]^{-1} \quad \text { and } \quad G^{\prime}(s)=F(s) \quad \forall s \in \mathbb{R} .
$$

Hence it follows from (A1) that $G$ is strictly convex, i.e.

$$
G(a)-G(c)<F(a)(a-c) \quad \forall a, c \in \mathbb{R}, \quad a \neq c .
$$

We note also for later purposes that

$$
\begin{aligned}
(F(c)-F(a)) c & \geq G(c)-F(a) c \\
& \geq \frac{c^{2}}{2 M_{1}}-\frac{|a||c|}{M_{0}} \geq \frac{c^{2}}{4 M_{1}}-\frac{M_{1} a^{2}}{M_{0}^{2}} \quad \forall a, c \in \mathbb{R} .
\end{aligned}
$$

Next, for any $r>1$, we regularise the non-differentiable nonlinearity $|\cdot|$ by the strictly convex functional $\frac{1}{r}|\cdot|_{r}$. We note for all $\underline{a}, \underline{c} \in \mathbb{R}^{2}$ that

$$
\begin{aligned}
& \frac{1}{r} \frac{\partial|\underline{a}|^{r}}{\partial a_{i}}=|\underline{a}|^{r-2} a_{i} \Rightarrow|\underline{a}|^{r-2} \underline{a} \cdot(\underline{a}-\underline{c}) \geq \frac{1}{r}\left[|\underline{a}|^{r}-|\underline{c}|^{r}\right], \\
&\left(|\underline{a}|^{r-2} \underline{a}-|\underline{c}|^{r-2} \underline{c}\right) \cdot \underline{a} \geq \frac{r-1}{r}\left[|\underline{a}|^{r}-|\underline{c}|^{r}\right] .
\end{aligned}
$$

In addition, for $r \in(1,2]$ we have that

$$
\left.|| \underline{a}\right|^{r-2} \underline{a}-|\underline{c}|^{r-2} \underline{c}\left|\leq\left(1+2^{2(2-r)}\right)^{\frac{1}{2}}\right| \underline{a}-\underline{c} \mid \quad \forall \underline{a}, \underline{c} \in \mathbb{R}^{2},
$$

see e.g. Lemma 1 in Chow $^{13}$.

For a given $r>1$, we then consider the following regularization of (Q):

$\left(\mathbf{Q}_{r}\right)$ Find $\underline{q}_{r} \in L^{2}\left(0, T ; \underline{V}^{r}(\Omega)\right)$ and $b_{r} \in H^{1}\left(0, T ; L^{2}(\Omega)\right)$ such that $b_{r}(\cdot, 0)=b^{0}(\cdot)$ and

$$
\begin{aligned}
\int_{0}^{T}\left(\partial_{t} b_{r}-\underline{\nabla} \cdot \underline{q}_{r}, \eta\right) \mathrm{d} t=0 & \forall \eta \in L^{2}\left(\Omega_{T}\right), \quad \text { (2.7a) } \\
\int_{0}^{T}\left[\left(k\left|\underline{q}_{r}\right|^{r-2} \underline{q}_{r}, \underline{v}\right)+\left(F\left(b_{r}\right)-F\left(b_{e}\right), \underline{\nabla} \cdot \underline{v}\right)\right] \mathrm{d} t=0 & \forall \underline{v} \in L^{2}\left(0, T ; \underline{V}^{r}(\Omega)\right) .
\end{aligned}
$$

For ease of exposition, we shall assume that $\Omega$ is a polygonal domain to avoid perturbation of domain errors in the finite element approximation. We make the following assumption

(A2) $\Omega$ is polygonal. Let $\left\{\mathcal{T}^{h}\right\}_{h>0}$ be a regular family of partitionings of $\Omega$ into disjoint open triangles $\sigma$ with $h_{\sigma}:=\operatorname{diam}(\sigma)$ and $h:=\max _{\sigma \in \mathcal{T}^{h}} h_{\sigma}$, so that $\bar{\Omega}=$ $\cup_{\sigma \in \mathcal{T}^{h}} \bar{\sigma}$.

Let $\underline{\nu}_{\partial \sigma}$ be the outward unit normal to $\partial \sigma$, the boundary of $\sigma$. We then introduce the lowest order Raviart-Thomas finite element spaces

$$
\begin{array}{ll}
\underline{V}^{h}:=\left\{\underline{v}^{h} \in\left[L^{\infty}(\Omega)\right]^{2}:\left.\underline{v}^{h}\right|_{\sigma}=\underline{a}_{\sigma}+c_{\sigma} \underline{x}, \underline{a}_{\sigma} \in \mathbb{R}^{2}, c_{\sigma} \in \mathbb{R}\right. & \left.\forall \sigma \in \mathcal{T}^{h}\right\} \subset \underline{V}^{\infty}(\Omega), \\
S^{h}:=\left\{\eta^{h} \in L^{\infty}(\Omega):\left.\eta^{h}\right|_{\sigma}=y_{\sigma} \in \mathbb{R} \quad \forall \sigma \in \mathcal{T}^{h}\right\} .
\end{array}
$$


Here the constraint $\underline{V}^{h} \subset \underline{V}^{\infty}(\Omega)$ yields for all $\underline{v} \in \underline{V}^{h}$ and for all adjacent triangles $\sigma, \sigma^{\prime} \in \mathcal{T}^{h}$ that

$$
\left.\underline{v}^{h}\right|_{\sigma} \cdot \underline{\nu}_{\partial \sigma}+\left.\underline{v}^{h}\right|_{\sigma^{\prime}} \cdot \underline{\nu}_{\partial \sigma^{\prime}}=0 \quad \text { on } \partial \sigma \cap \partial \sigma^{\prime} .
$$

Let $P^{h}: L^{1}(\Omega) \rightarrow S^{h}$ be such that

$$
\left(\left(I-P^{h}\right) z, \eta^{h}\right)=0 \quad \forall \eta^{h} \in S^{h} .
$$

It follows for all $s \in[1, \infty]$, that if $z \in L^{s}(\Omega)$ then

$$
\left|P^{h} z\right|_{0, s, \Omega} \leq|z|_{0, s, \Omega} \quad \text { and } \quad \lim _{h \rightarrow 0}\left|\left(I-P^{h}\right) z\right|_{0, s, \Omega}=0 .
$$

In addition, let $0=t_{0}<t_{1}<\ldots<t_{N-1}<t_{N}=T$ be a partitioning of $[0, T]$ into possibly variable time steps $\tau_{n}:=t_{n}-t_{n-1}, n=1 \rightarrow N$. We set $\tau:=$ $\max _{n=1 \rightarrow N} \tau_{n}$ and

$$
b_{e}^{n}:=b_{e}\left(t_{n}\right) \quad n=0 \rightarrow N .
$$

Our fully practical approximation of $\left(\mathrm{Q}_{r}\right)$ by $\underline{V}^{h}$ is then: $\left(\mathbf{Q}_{r}^{h, \tau}\right)$ For $n \geq 1$, find $\underline{Q}_{r}^{n} \in \underline{V}^{h}$ and $B_{r}^{n} \in S^{h}$ such that

$$
\begin{aligned}
\left(B_{r}^{n}-\tau_{n} \underline{\nabla} \cdot \underline{Q}_{r}^{n}, \eta^{h}\right) & =\left(B_{r}^{n-1}, \eta^{h}\right) & & \forall \eta^{h} \in S^{h}, \\
\left(k\left|\underline{Q}_{r}^{n}\right|^{r-2} \underline{Q}_{r}^{n}, \underline{v}^{h}\right)^{h}+\left(F\left(B_{r}^{n}\right)-F\left(b_{e}^{n}\right), \underline{\nabla} \cdot \underline{v}^{h}\right) & =0 & & \forall \underline{v}^{h} \in \underline{V}^{h} ;
\end{aligned}
$$

where $B_{r}^{0}=F^{-1}\left(P^{h} F\left(b^{0}\right)\right)$.

Here $(\underline{v}, \underline{z})^{h}:=\sum_{\sigma \in \mathcal{T}^{h}}(\underline{v}, \underline{z})_{\sigma}^{h}$, and

$$
(\underline{v}, \underline{z})_{\sigma}^{h}:=\frac{1}{3}|\sigma| \sum_{j=1}^{3} \underline{v}\left(P_{j}^{\sigma}\right) \cdot \underline{z}\left(P_{j}^{\sigma}\right) \quad \forall \underline{v}, \underline{z} \in[C(\bar{\sigma})]^{2}, \quad \forall \sigma \in \mathcal{T}^{h} ;
$$

where $\left\{P_{j}^{\sigma}\right\}_{j=1}^{3}$ are the vertices of $\sigma$. Hence $(\underline{v}, \underline{z})^{h}$ averages the integrand $\underline{v} \cdot \underline{z}$ over each triangle $\sigma$ at its vertices and is exact if $\underline{v} \cdot \underline{z}$ is piecewise linear over the partitioning $\mathcal{T}^{h}$. For any $r \geq 1$ and for any $\underline{v}^{h} \in \underline{V}^{h}$, we have from the equivalence of norms for $\underline{v}^{h}$ and the convexity of $|\cdot|^{r}$ that

$$
C_{r}\left(\left|\underline{v}^{h}\right|^{r}, 1\right)_{\sigma}^{h} \leq \int_{\sigma}\left|\underline{v}^{h}\right|^{r} \mathrm{~d} \underline{x} \leq\left(\left|\underline{v}^{h}\right|^{r}, 1\right)_{\sigma}^{h} \equiv \frac{1}{3}|\sigma| \sum_{j=1}^{3}\left|\underline{v}^{h}\left(P_{j}^{\sigma}\right)\right|^{r} \quad \forall \sigma \in \mathcal{T}^{h} .
$$

It follows from (2.15) that

$$
\begin{aligned}
\left(k\left|\underline{v}^{h}\right|^{r}, 1\right)^{h} & =\sum_{\sigma \in \mathcal{T}^{h}}\left[\left(\left[P^{h} k\right]\left|\underline{v}^{h}\right|^{r}, 1\right)^{h}+\left(\left(I-P^{h}\right) k\left|\underline{v}^{h}\right|^{r}, 1\right)^{h}\right] \\
& \geq \sum_{\sigma \in \mathcal{T}^{h}}\left(\left[P^{h} k\right]\left|\underline{v}^{h}\right|^{r}, 1\right)-\left|\left(I-P^{h}\right) k\right|_{0, \infty, \Omega}\left(\left|\underline{v}^{h}\right|^{r}, 1\right)^{h} \\
& \geq\left(k\left|\underline{v}^{h}\right|^{r}, 1\right)-C\left|\left(I-P^{h}\right) k\right|_{0, \infty, \Omega}\left|\underline{v}^{h}\right|_{0, r, \Omega}^{r} \quad \forall \underline{v}^{h} \in \underline{V}^{h} .
\end{aligned}
$$


We note from the definition of $B_{r}^{0},(2.2),(2.11)$ and (A1) that for all $s \in[1, \infty]$

$$
\begin{aligned}
\left|B_{r}^{0}\right|_{0, s, \Omega} & =\left|F^{-1}\left(P^{h} F\left(b^{0}\right)\right)\right|_{0, s, \Omega} \leq M_{1}\left|P^{h} F\left(b^{0}\right)\right|_{0, s, \Omega} \leq M_{1}\left|F\left(b^{0}\right)\right|_{0, s, \Omega} \\
& \leq \frac{M_{1}}{M_{0}}\left|b^{0}\right|_{0, s, \Omega} \leq C .
\end{aligned}
$$

Theorem 2.1. Let the Assumptions (A1) and (A2) hold. Then for all $r>1$, for all regular partitionings $\mathcal{T}^{h}$ of $\Omega$, and for all $\tau_{n}>0$, there exists a unique solution, $\underline{Q}_{r}^{n} \in \underline{V}^{h}$ and $B_{r}^{n} \in S^{h}$, to the $n^{\text {th }}$ step of $\left(Q_{r}^{h, \tau}\right)$. Moreover, we have that

$$
\begin{gathered}
\max _{n=0 \rightarrow N}\left|B_{r}^{n}\right|_{0, \Omega}+\max _{n=0 \rightarrow N}\left|F\left(B_{r}^{n}\right)\right|_{0, \Omega}+\sum_{n=1}^{N} \tau_{n}\left(k\left|\underline{Q}_{r}^{n}\right|^{r}, 1\right)^{h} \leq C(T), \\
\frac{r-1}{r} \max _{n=1 \rightarrow N}\left(k\left|\underline{Q}_{n}^{n}\right|^{r}, 1\right)^{h}+\sum_{n=1}^{N} \tau_{n}\left|\frac{B_{r}^{n}-B_{r}^{n-1}}{\tau_{n}}\right|_{0, \Omega}^{2}+\sum_{n=1}^{N} \tau_{n}\left|\underline{\nabla} \cdot \underline{Q}_{r}^{n}\right|_{0, \Omega}^{2} \leq C .
\end{gathered}
$$

Proof. It follows from (2.13a) that

$$
B_{r}^{n}=B_{r}^{n-1}+\tau_{n} \underline{\nabla} \cdot \underline{Q}_{r}^{n} .
$$

On noting this, (2.5a) and (2.3), we see that (2.13b), on noting (2.13a), is the Euler-Lagrange equation for the strictly convex minimization problem

$$
\min _{\underline{v}^{h} \in \underline{V}^{h}} \mathcal{E}_{r}^{n}\left(\underline{v}^{h}, B_{r}^{n-1}+\tau_{n} \underline{\nabla} \cdot \underline{v}^{h}\right),
$$

where

$$
\begin{array}{r}
\mathcal{E}_{r}^{n}\left(\underline{v}^{h}, \eta^{h}\right):=\frac{\tau_{n}}{r}\left(k\left|\underline{v}^{h}\right|^{r}, 1\right)^{h}-\tau_{n} F\left(b_{e}^{n}\right)\left(\underline{\nabla} \cdot \underline{v}^{h}, 1\right)+\left(G\left(\eta^{h}\right), 1\right) \\
\forall \underline{v}^{h} \in \underline{V}^{h}, \quad \forall \eta^{h} \in S^{h} .
\end{array}
$$

Hence the desired existence and uniqueness results for $\underline{Q}_{r}^{n}$ and $B_{r}^{n}$ immediately follow from this and (2.19).

Choosing $\eta^{h} \equiv F\left(B_{r}^{n}\right)-F\left(b_{e}^{n}\right)$ in (2.13a) and $\underline{v}^{h} \equiv \underline{Q}_{r}^{n}$ in (2.13b), and combining yields that

$$
\tau_{n}\left(k\left|\underline{Q}_{r}^{n}\right|^{r}, 1\right)^{h}+\left(F\left(B_{r}^{n}\right)-F\left(b_{e}^{n}\right), B_{r}^{n}-B_{r}^{n-1}\right)=0 .
$$


We have from (2.21), (2.3), (2.2), (A1), (2.12) and (2.4) that

$$
\begin{aligned}
& \left(G\left(B_{r}^{n}\right)-F\left(b_{e}^{n}\right) B_{r}^{n}, 1\right)+\tau_{n}\left(k\left|\underline{Q}_{r}^{n}\right|^{r}, 1\right)^{h} \\
& \quad \leq\left(G\left(B_{r}^{n-1}\right)-F\left(b_{e}^{n-1}\right) B_{r}^{n-1}, 1\right)-\left(F\left(b_{e}^{n}\right)-F\left(b_{e}^{n-1}\right), B_{r}^{n-1}\right) \\
& \quad \leq\left(G\left(B_{r}^{n-1}\right)-F\left(b_{e}^{n-1}\right) B_{r}^{n-1}, 1\right)+M_{0}^{-1} \tau_{n}\left|\frac{b_{e}^{n}-b_{e}^{n-1}}{\tau_{n}}\right|_{0, \Omega}\left|B_{r}^{n-1}\right|_{0, \Omega} \\
& \quad \leq\left(G\left(B_{r}^{n-1}\right)-F\left(b_{e}^{n-1}\right) B_{r}^{n-1}, 1\right)+\frac{\tau_{n}}{4 M_{1}}\left|B_{r}^{n-1}\right|_{0, \Omega}^{2}+\frac{M_{1}|\Omega|}{M_{0}^{2}} \int_{t_{n-1}}^{t_{n}}\left|d_{t} b_{e}\right|^{2} \mathrm{~d} t \\
& \quad \leq\left(1+\tau_{n}\right)\left(G\left(B_{r}^{n-1}\right)-F\left(b_{e}^{n-1}\right) B_{r}^{n-1}, 1\right)+\frac{M_{1}|\Omega|}{M_{0}^{2}} \int_{t_{n-1}}^{t_{n}}\left[\left|b_{e}^{n-1}\right|^{2}+\left|d_{t} b_{e}\right|^{2}\right] \mathrm{d} t .
\end{aligned}
$$

It follows immediately from (2.22) that for $n=1 \rightarrow N$

$$
\left(G\left(B_{r}^{n}\right)-F\left(b_{e}^{n}\right) B_{r}^{n}, 1\right) \leq e^{t_{n}}\left[\left(G\left(B_{r}^{0}\right)-F\left(b_{e}^{0}\right) B_{r}^{0}, 1\right)+C \int_{0}^{t_{n}}\left[\left|b_{e}\right|^{2}+\left|d_{t} b_{e}\right|^{2}\right] \mathrm{d} t\right] .
$$

In addition, we have from (2.4), (2.10), (A1) and (2.17) that

$$
\begin{aligned}
\left(G\left(B_{r}^{0}\right)-F\left(b_{e}^{0}\right) B_{r}^{0}, 1\right) & \leq\left(F\left(B_{r}^{0}\right)-F\left(b_{e}^{0}\right), B_{r}^{0}\right)=\left(F\left(b^{0}\right)-F\left(b_{e}^{0}\right), B_{r}^{0}\right) \\
& \leq\left[\left|F\left(b^{0}\right)\right|_{0, \infty, \Omega}+\left|F\left(b_{e}^{0}\right)\right|\right]\left|B_{r}^{0}\right|_{0,1, \Omega} \leq C .
\end{aligned}
$$

The first two bounds in the desired result (2.18a) then follow from (2.23), (2.24), (2.4), (2.2) and (A1).

Summing (2.22) from $n=1$ to $N$ yields, on noting (2.23), (2.24) and (A1), that

$$
\left(G\left(B_{r}^{N}\right)-F\left(b_{e}^{N}\right) B_{r}^{N}, 1\right)+\sum_{n=1}^{N} \tau_{n}\left(k\left|\underline{Q}_{r}^{n}\right|^{r}, 1\right)^{h} \leq C(T) .
$$

Hence the third bound in (2.18a) follows from (2.25), (2.4) and (A1).

Choosing $\eta^{h} \equiv \frac{\left[F\left(B_{r}^{n}\right)-F\left(B_{r}^{n-1}\right)\right]-\left[F\left(b_{e}^{n}\right)-F\left(b_{e}^{n-1}\right)\right]}{\tau_{n}}$ in (2.13a), and noting (2.13b) and (2.5b), yields for $n=2 \rightarrow N$ that

$$
\begin{aligned}
\tau_{n}\left(\frac{B_{r}^{n}-B_{r}^{n-1}}{\tau_{n}},\right. & \left.\frac{\left[F\left(B_{r}^{n}\right)-F\left(B_{r}^{n-1}\right)\right]-\left[F\left(b_{e}^{n}\right)-F\left(b_{e}^{n-1}\right)\right]}{\tau_{n}}\right) \\
& \left.=\left(\underline{\nabla} \cdot \underline{Q}_{r}^{n}, F\left(B_{r}^{n}\right)-F\left(B_{r}^{n-1}\right)\right]-\left[F\left(b_{e}^{n}\right)-F\left(b_{e}^{n-1}\right)\right]\right) \\
& =-\left(k\left[\left|\underline{Q}_{r}^{n}\right|^{r-2} \underline{Q}_{r}^{n}-\left|\underline{Q}_{r}^{n-1}\right|^{r-2} \underline{Q}_{r}^{n-1}\right], \underline{Q}_{r}^{n}\right)^{h} \\
& \leq-\frac{r-1}{r}\left(k\left[\left|\underline{Q}_{r}^{n}\right|^{r}-\left|\underline{Q}_{r}^{n-1}\right|^{r}\right], 1\right)^{h} ;
\end{aligned}
$$

and

$$
\begin{aligned}
\tau_{1}\left(\frac{B_{r}^{1}-B_{r}^{0}}{\tau_{1}}, \frac{\left[F\left(B_{r}^{1}\right)-F\left(B_{r}^{0}\right)\right]-\left[F\left(b_{e}^{1}\right)-F\left(b_{e}^{0}\right)\right]}{\tau_{1}}\right) \\
=\left(\underline{\nabla} \cdot \underline{Q}_{r}^{1},\left[F\left(B_{r}^{1}\right)-F\left(B_{r}^{0}\right)\right]-\left[F\left(b_{e}^{1}\right)-F\left(b_{e}^{0}\right)\right]\right) \\
=-\left(k\left|\underline{Q}_{r}^{1}\right|^{r}, 1\right)^{h}-\left(\underline{\nabla} \cdot \underline{Q}_{r}^{1}, F\left(B_{r}^{0}\right)-F\left(b_{e}^{0}\right)\right) .
\end{aligned}
$$


Next it follows, as $W_{r}^{0}=F^{-1}\left(P^{h} F\left(w^{0}\right)\right), \underline{\nabla} \cdot \underline{v}^{h} \in S^{h}$ for all $\underline{v}^{h} \in \underline{V}^{h}$ and on noting (A1) and (2.15), that

$$
\begin{aligned}
-\left(\underline{\nabla} \cdot \underline{Q}_{r}^{1}, F\left(B_{r}^{0}\right)-F\left(b_{e}^{0}\right)\right) & =-\left(\underline{\nabla} \cdot \underline{Q}_{r}^{1}, F\left(b^{0}\right)-F\left(b_{e}^{0}\right)\right)=\left(\underline{Q}_{r}^{1}, \underline{\nabla}\left[F\left(b^{0}\right)\right]\right) \\
& \leq\left(k,\left|\underline{Q}_{r}^{1}\right|\right) \leq \frac{1}{r}\left(k\left|\underline{Q}_{r}^{1}\right|^{r}, 1\right)+\frac{r-1}{r}(k, 1)^{h} \\
& \leq \frac{1}{r}\left(k\left|\underline{Q}_{r}^{1}\right|^{r}, 1\right)^{h}+C .
\end{aligned}
$$

Summing (2.26a), including (2.26b) and noting (2.27) yields for $n=1 \rightarrow N$ that

$$
\begin{aligned}
\frac{r-1}{r}\left(k\left|\underline{Q}_{r}^{n}\right|^{r}, 1\right)^{h} & +\sum_{\ell=1}^{n} \tau_{\ell}\left(\frac{B_{r}^{\ell}-B_{r}^{\ell-1}}{\tau_{\ell}}, \frac{F\left(B_{r}^{\ell}\right)-F\left(B_{r}^{\ell-1}\right)}{\tau_{\ell}}\right) \\
& \leq C+\sum_{\ell=1}^{n} \tau_{\ell}\left(\frac{B_{r}^{\ell}-B_{r}^{\ell-1}}{\tau_{\ell}}, \frac{F\left(b_{e}^{\ell}\right)-F\left(b_{e}^{\ell-1}\right)}{\tau_{\ell}}\right) .
\end{aligned}
$$

The first two bounds in the desired result (2.18b) then follow from (2.28), (2.2), (2.12) and (A1), on using a Young's inequality. Finally the third bound in (2.18b) then follows from the second bound in (2.18b) and (2.19).

\section{Convergence of $\left(\mathrm{Q}_{r}^{h, \tau}\right)$ - Existence Theory for (Q)}

As the stability bounds (2.18a,b) do not control spatial derivatives of $\left\{\underline{Q}_{r}^{n}, B_{r}^{n}\right\}_{n=1}^{N}$, except for $\left\{\underline{\nabla} \cdot \underline{Q}_{r}^{n}\right\}_{n=1}^{N}$; we cannot exploit compactness to get strong convergence of $\left\{B_{r}^{n}\right\}_{n=1}^{N}$, which we require to pass to the limit in $F\left(B_{r}^{n}\right)$ in $\left(\mathrm{Q}_{r}^{h, \tau}\right)$. Hence we prove the subsequence convergence of solutions to $\left(\mathrm{Q}_{r}^{h, \tau}\right),(2.13 \mathrm{a}, \mathrm{b})$, to solutions of $(Q),(1.15 \mathrm{a}, \mathrm{b})$, as $h, \tau \rightarrow 0$ and $r \rightarrow 1$ in stages. First, we introduce the following intermediate problem, a discrete in time approximation of $\left(\mathrm{Q}_{r}\right)$ for $r>1$ :

$\left(\mathbf{Q}_{r}^{\tau}\right)$ For $n \geq 1$, find $\underline{q}_{r}^{n} \in \underline{V}^{r}(\Omega)$ and $b_{r}^{n} \in L^{2}(\Omega)$ such that

$$
\begin{aligned}
\left(b_{r}^{n}-\tau_{n} \underline{\nabla} \cdot \underline{q}_{r}^{n}, \eta\right) & =\left(b_{r}^{n-1}, \eta\right) & & \forall \eta \in L^{2}(\Omega), \\
\left(k\left|\underline{q}_{r}^{n}\right|^{r-2} \underline{q}_{r}^{n}, \underline{v}\right)+\left(F\left(b_{r}^{n}\right)-F\left(b_{e}^{n}\right), \underline{\nabla} \cdot \underline{v}\right) & =0 & & \forall \underline{v} \in \underline{V}^{r}(\Omega) ;
\end{aligned}
$$

where $b_{r}^{0}=b^{0}$.

We then show that the unique solution of $\left(\mathrm{Q}_{r}^{h, \tau}\right)$ converges to the unique solution of $\left(\mathrm{Q}_{r}^{\tau}\right)$ as $h \rightarrow 0$, by exploiting the monotonicity result (2.5a) and the monotonicity of $F$. In order to achieve this we introduce the generalised interpolation operator $\underline{I}^{h}: \underline{V}^{r}(\Omega) \cap\left[W^{1, r}(\Omega)\right]^{n} \rightarrow \underline{V}^{h}$, where $r>1$, satisfying

$$
\int_{\partial_{i} \sigma}\left(\underline{v}-\underline{I}^{h} \underline{v}\right) \cdot \underline{\nu}_{\partial_{i} \sigma} d s=0 \quad i=1 \rightarrow 3, \quad \forall \sigma \in \mathcal{T}^{h} ;
$$

where $\partial \sigma \equiv \cup_{i=1}^{3} \partial_{i} \sigma$ and $\underline{\nu}_{\partial_{i} \sigma}$ are the corresponding outward unit normals on $\partial_{i} \sigma$. It follows that

$$
\left(\underline{\nabla} \cdot\left(\underline{v}-\underline{I}^{h} \underline{v}\right), \eta^{h}\right)=0 \quad \forall \eta^{h} \in S^{h} .
$$

In addition, we have for all $\sigma \in \mathcal{T}^{h}$ and any $s \in(1, \infty]$ that

$$
\left|\underline{v}-\underline{I}^{h} \underline{v}\right|_{0, s, \sigma} \leq C_{s} h_{\sigma}|\underline{v}|_{1, s, \sigma} \quad \text { and } \quad\left|\underline{I}^{h} \underline{v}\right|_{1, s, \sigma} \leq C_{s}|\underline{v}|_{1, s, \sigma},
$$


e.g. see Lemma 3.1 in Farhloul ${ }^{15}$ and the proof given there for $s \geq 2$ is also valid for any $s \in(1, \infty)$. Furthermore, it follows from (2.5a), (2.14) and (3.4) for any $r>1$ and any $\sigma \in \mathcal{T}^{h}$ that

$$
\begin{aligned}
\left.\left|\int_{\sigma}\right| \underline{I}^{h} \underline{v}\right|^{r}-\left.\left(\left|\underline{I}^{h} \underline{v}\right|^{r}, 1\right)_{\sigma}^{h}\left|\leq C h_{\sigma}\right| \sigma||\left[\underline{I}^{h} \underline{v}\right]^{r}\right|_{1, \infty, \sigma} \leq C r h_{\sigma}|\sigma|\|\underline{v}\|_{1, \infty, \sigma}^{r} \\
\forall \underline{v} \in\left[W^{1, \infty}(\sigma)\right]^{2} .
\end{aligned}
$$

Hence, similarly to (2.16), it follows from (3.5) and (3.4) that

$$
\begin{array}{r}
\left|\left(k\left|\underline{I}^{h} \underline{v}\right|^{r}, 1\right)-\left(k \mid \underline{I}^{h} \underline{v}^{r}, 1\right)^{h}\right| \leq C\left[r k_{1}+\left|\left(I-P^{h}\right) k\right|_{0, \infty, \Omega}\right]\|\underline{v}\|_{1, \infty, \sigma}^{r} \\
\forall \underline{v} \in\left[W^{1, \infty}(\Omega)\right]^{2} .
\end{array}
$$

Theorem 3.1. Let the Assumptions (A1) and (A2) hold. For all regular partitionings $\mathcal{T}^{h}$ of $\Omega$, and for all time partitions $\left\{\tau_{n}\right\}_{n=1}^{N}$ and for all $r>1$ the unique solution $\left\{\underline{Q}_{r}^{n}, B_{r}^{n}\right\}_{n=1}^{N}$ to $\left(Q_{r}^{h, \tau}\right)$ is such that as $h \rightarrow 0$

$$
\begin{aligned}
& \underline{Q}_{r}^{n} \rightarrow \underline{q}_{r}^{n} \quad \text { weakly in }\left[L^{r}(\Omega)\right]^{2}, \quad n=1 \rightarrow N, \\
& \underline{\nabla} \cdot \underline{Q}_{r}^{n} \rightarrow \underline{\nabla} \cdot \underline{q}_{r}^{n} \quad \text { weakly in } L^{2}(\Omega), \quad n=1 \rightarrow N, \\
& B_{r}^{n} \rightarrow b_{r}^{n} \quad \text { strongly in } L^{2}(\Omega), \quad n=0 \rightarrow N \text {, } \\
& F\left(B_{r}^{n}\right) \rightarrow F\left(b_{r}^{n}\right) \quad \text { strongly in } L^{2}(\Omega), \quad n=0 \rightarrow N ;
\end{aligned}
$$

where $\left\{\underline{q}_{r}^{n}, b_{r}^{n}\right\}_{n=1}^{N}$ is the unique solution of $\left(Q_{r}^{\tau}\right),(3.1 a, b)$.

Moreover, we have that

$$
\begin{gathered}
\frac{r-1}{r} \max _{n=1 \rightarrow N}\left(k\left|\underline{q}_{r}^{n}\right|^{r}, 1\right)+\sum_{n=1}^{N} \tau_{n}\left|\underline{\nabla} \cdot \underline{q}_{r}^{n}\right|_{0, \Omega}^{2}+\max _{n=0 \rightarrow N}\left|b_{r}^{n}\right|_{0, \Omega}+\max _{n=0 \rightarrow N}\left|F\left(b_{r}^{n}\right)\right|_{0, \Omega} \\
+\sum_{n=1}^{N} \tau_{n}\left|\frac{b_{r}^{n}-b_{r}^{n-1}}{\tau_{n}}\right|_{0, \Omega}^{2}+\max _{n=1 \rightarrow N}\left|\nabla b_{r}^{n}\right|_{0, p, \Omega} \leq C(T)
\end{gathered}
$$

where $p=\frac{r}{r-1}$.

Proof. It follows immediately from the bounds (2.18a,b), (2.15), our assumptions on $k$ and (2.5a) that there exist $\left\{\underline{q}_{r}^{n}\right\}_{n=1}^{N}$ and $\left\{b_{r}^{n}\right\}_{n=0}^{N}$ such that $(3.7 \mathrm{a}, \mathrm{b})$ hold for a subsequence $\left\{\left\{\underline{Q}_{r}^{n}, B_{r}^{n}\right\}_{n=1}^{N}\right\}_{h_{j}>0}$ of $\left\{\left\{\underline{Q}_{r}^{n}, B_{r}^{n}\right\}_{n=1}^{N}\right\}_{h>0}$, the bounds on $\left\{\underline{q}_{r}^{n}\right\}_{n=1}^{N}$ hold in (3.8) and there exist $\left\{b_{r}^{n}, g_{r}^{n}\right\}_{n=1}^{N}$ such that as $h_{j} \rightarrow 0$

$$
B_{r}^{n} \rightarrow b_{r}^{n}, \quad F\left(B_{r}^{n}\right) \rightarrow g_{r}^{n} \quad \text { weakly in } L^{2}(\Omega), \quad n=1 \rightarrow N .
$$

It follows from (2.11) and (2.2) that as $h \rightarrow 0$

$$
F\left(B_{r}^{0}\right) \rightarrow F\left(b^{0}\right) \equiv F\left(b_{r}^{0}\right), \quad B_{r}^{0} \rightarrow b^{0} \equiv b_{r}^{0} \quad \text { strongly in } L^{\infty}(\Omega) .
$$

We now show that $\left\{\underline{q}_{r}^{n}, b_{r}^{n}\right\}_{n=1}^{N}$ is a solution of $\left(\mathrm{Q}_{r}^{\tau}\right)$. For any $\eta \in L^{2}(\Omega)$, we choose $\eta^{h} \equiv P^{h} \eta \in S^{h}$ in the $h_{j}$ version of (2.13a). We can now pass to the limit $h_{j} \rightarrow 0$ in this, and obtain, on noting (3.7b), (3.9), and (2.11), the desired result (3.1a) for $n=1 \rightarrow N$. 
It follows from $(2.13 \mathrm{a}, \mathrm{b}),(2.5 \mathrm{a})$, the monotonicity of $F$ and (2.16) that for any $\underline{v}^{h} \in \underline{V}^{h}$ and $\eta^{h} \in S^{h}$

$$
\begin{aligned}
\tau_{n}^{-1} & \left(F\left(B_{r}^{n}\right)-F\left(b_{e}^{n}\right), B_{r}^{n-1}-\eta^{h}+\tau_{n} \underline{\nabla} \cdot \underline{v}^{h}\right) \\
= & \tau_{n}^{-1}\left(F\left(B_{r}^{n}\right)-F\left(b_{e}^{n}\right), B_{r}^{n}-\eta^{h}-\tau_{n} \underline{\nabla} \cdot\left(\underline{Q}_{r}^{n}-\underline{v}^{h}\right)\right) \\
= & \left(k\left|\underline{Q}_{r}^{n}\right|^{r-2} \underline{Q}_{r}^{n}, \underline{Q}_{r}^{n}-\underline{v}^{h}\right)^{h}+\tau_{n}^{-1}\left(F\left(B_{r}^{n}\right)-F\left(b_{e}^{n}\right), B_{r}^{n}-\eta^{h}\right) \\
\geq & r^{-1}\left(k\left[\left|\underline{Q}_{r}^{n}\right|^{r}-\left|\underline{v}^{h}\right|^{r}\right], 1\right)^{h}+\tau_{n}^{-1}\left(F\left(\eta^{h}\right)-F\left(b_{e}^{n}\right), B_{r}^{n}-\eta^{h}\right) \\
\geq & \left(k\left|\underline{v}^{h}\right|^{r-2} \underline{v}^{h}, \underline{Q}_{r}^{n}-\underline{v}^{h}\right)+r^{-1}\left[\left(k\left|\underline{v}^{h}\right|^{r}, 1\right)-\left(k\left|\underline{v}^{h}\right|^{r}, 1\right)^{h}\right] \\
& \quad+\tau_{n}^{-1}\left(F\left(\eta^{h}\right)-F\left(b_{e}^{n}\right), B_{r}^{n}-\eta^{h}\right)-C r^{-1}\left|\left(I-P^{h}\right) k\right|_{0, \infty, \Omega}\left|\underline{Q}_{r}^{n}\right|_{0, r, \Omega}^{r} .
\end{aligned}
$$

For any $\underline{v} \in\left[C^{\infty}(\bar{\Omega})\right]^{2}$ and $\eta \in L^{2}(\Omega)$, we choose $\underline{v}^{h} \equiv \underline{I}^{h} \underline{v}$ and $\eta^{h} \equiv P^{h} \eta$ in the $h_{j}$ version of (3.11) with $n=\ell$, for some integer $\ell \in[1, N]$. Assuming that $B_{r}^{\ell-1} \rightarrow b_{r}^{\ell-1}$ strongly in $L^{2}(\Omega)$ as $h_{j} \rightarrow 0$, we can now pass to the limit $h_{j} \rightarrow 0$ in this, and obtain, on noting (3.1a), (3.7a), (3.9), (2.11), (3.3), (2.6), (3.4) and (3.6) that

$$
\begin{aligned}
\tau_{\ell}^{-1}\left(g_{r}^{\ell}-F\left(b_{e}^{\ell}\right),\right. & \left.b_{r}^{\ell}-\eta-\tau_{\ell} \underline{\nabla} \cdot\left(\underline{q}_{r}^{\ell}-\underline{v}\right)\right) \\
& =\tau_{\ell}^{-1}\left(g_{r}^{\ell}-F\left(b_{e}^{\ell}\right), b_{r}^{\ell-1}-\eta+\tau_{\ell} \underline{\nabla} \cdot \underline{v}\right) \\
\geq\left(k|\underline{v}|^{r-2} \underline{v}, \underline{q}_{r}^{\ell}-\underline{v}\right)+\tau_{\ell}^{-1}\left(F(\eta)-F\left(b_{e}^{\ell}\right), b_{r}^{\ell}-\eta\right) & \forall\{\underline{v}, \eta\} \in\left[C^{\infty}(\bar{\Omega})\right]^{2} \times L^{2}(\Omega) .
\end{aligned}
$$

As $\left[C^{\infty}(\bar{\Omega})\right]^{2}$ is dense in $\underline{V}^{r}(\Omega)$, recall $(1.21)$, and $\left\{\underline{q}_{r}^{\ell}, b_{r}^{\ell}\right\} \in \underline{V}^{r}(\Omega) \times L^{2}(\Omega)$, it follows that (3.12) holds for all $\{\underline{v}, \eta\} \in \underline{V}^{r}(\Omega) \times L^{2}(\Omega)$. For any fixed $\underline{z} \in \underline{V}^{r}(\Omega)$ and $\lambda \in \mathbb{R}_{>0}$, choosing $\underline{v} \equiv \underline{q}_{r}^{\ell} \pm \lambda \underline{z}$ and $\eta \equiv b_{r}^{\ell}$ in (3.12) yields that

$$
\mp\left[\left(k\left|\underline{q}_{r}^{\ell} \pm \lambda \underline{z}\right|^{r-2}\left(\underline{q}_{r}^{\ell} \pm \lambda \underline{z}\right), \underline{z}\right)+\left(g_{r}^{\ell}-F\left(b_{e}^{\ell}\right), \underline{\nabla} \cdot \underline{z}\right)\right] \geq 0 .
$$

Sending $\lambda \rightarrow 0$, and repeating the above for all $\underline{z} \in \underline{V}^{r}(\Omega)$ yields that

$$
\left(k\left|\underline{q}_{r}^{\ell}\right|^{r-2} \underline{q}_{r}^{\ell}, \underline{v}\right)+\left(g_{r}^{\ell}-F\left(b_{e}^{\ell}\right), \underline{\nabla} \cdot \underline{v}\right)=0 \quad \forall \underline{v} \in \underline{V}^{r}(\Omega) .
$$

For any fixed $\varphi \in L^{2}(\Omega)$ and $\lambda \in \mathbb{R}_{>0}$, choosing $\underline{v} \equiv \underline{q}_{r}^{\ell}$ and $\eta \equiv b_{r}^{\ell} \pm \lambda \varphi$ in (3.12) yields that

$$
\pm\left[\left(F\left(b_{r}^{\ell} \pm \lambda \varphi\right)-\left(g_{r}^{\ell}, \varphi\right)\right] \geq 0 .\right.
$$

Sending $\lambda \rightarrow 0$, and repeating the above for all $\varphi \in L^{2}(\Omega)$ yields that $g_{r}^{\ell}=F\left(b_{r}^{\ell}\right)$, and hence this and (3.14) yield that (3.1b) holds with $n=\ell$.

It is a simple matter to show that for a given $b_{r}^{\ell-1} \in L^{2}(\Omega)$ the solution $\left\{\underline{q}_{r}^{\ell}, b_{r}^{\ell}\right\}$ is unique. Hence the whole sequence converges in $(3.7 \mathrm{a}, \mathrm{b})$ and weakly in $(3.7 \mathrm{c}, \mathrm{d})$ for $n=\ell$.

To complete the induction step, we need to show that (3.7c) holds, and hence (3.7d), for $n=\ell$. From (3.1a) and (2.13a) with $n=\ell$ and $\eta \equiv \eta^{h} \equiv F\left(B_{r}^{\ell}\right)$, we have 
that

$$
\begin{aligned}
\left(b_{r}^{\ell}-B_{r}^{\ell}, F\left(b_{r}^{\ell}\right)-F\left(B_{r}^{\ell}\right)\right)= & \left(b_{r}^{\ell}-B_{r}^{\ell}, F\left(b_{r}^{\ell}\right)\right)-\left(b_{r}^{\ell-1}-B_{r}^{\ell-1}, F\left(B_{r}^{\ell}\right)\right) \\
& -\tau_{\ell}\left(\underline{\nabla} \cdot\left(\underline{q}_{r}^{\ell}-\underline{Q}_{r}^{\ell}\right),\left[F\left(B_{r}^{\ell}\right)-F\left(b_{e}^{\ell}\right)\right]+F\left(b_{e}^{\ell}\right)\right) .
\end{aligned}
$$

Next we note from (3.1b) and (2.13b) with $n=\ell$ and $\underline{v} \equiv \underline{q}_{r}^{\ell}$ and $\underline{v}^{h} \equiv \underline{Q}_{r}^{\ell}$ that

$$
\begin{aligned}
- & \left(\underline{\nabla} \cdot\left(\underline{q}_{r}^{\ell}-\underline{Q}_{r}^{\ell}\right), F\left(B_{r}^{\ell}\right)-F\left(b_{e}^{\ell}\right)\right) \\
& =-\left(\underline{\nabla} \cdot \underline{q}_{r}^{\ell}, F\left(B_{r}^{\ell}\right)-F\left(b_{r}^{\ell}\right)\right)-\left(\underline{\nabla} \cdot \underline{q}_{r}^{\ell}, F\left(b_{r}^{\ell}\right)-F\left(b_{e}^{\ell}\right)\right)+\left(\underline{\nabla} \cdot \underline{Q}_{r}^{\ell}, F\left(B_{r}^{\ell}\right)-F\left(b_{e}^{\ell}\right)\right) \\
& =-\left(\underline{\nabla} \cdot \underline{q}_{r}^{\ell}, F\left(B_{r}^{\ell}\right)-F\left(b_{r}^{\ell}\right)\right)+\left(k\left|\underline{q}_{r}^{\ell}\right|^{r}, 1\right)-\left(k\left|\underline{Q}_{r}^{\ell}\right|^{r}, 1\right)^{h} .
\end{aligned}
$$

In addition, we note from (2.16) and (2.5a) that

$$
\begin{aligned}
\left(k\left|\underline{q}_{r}^{\ell}\right|^{r}, 1\right)-\left(k\left|\underline{Q}_{r}^{\ell}\right|^{r}, 1\right)^{h} & \\
& \leq\left(k\left[\left|\underline{q}_{r}^{\ell}\right|^{r}-\left|\underline{Q}_{r}^{\ell}\right|^{r}\right], 1\right)+C\left|\left(I-P^{h}\right) k\right|_{0, \infty, \Omega}\left|\underline{Q}_{r}^{\ell}\right|_{0, r, \Omega}^{r} \\
& \leq r\left(k\left|\underline{q}_{r}^{\ell}\right|^{r-2} \underline{q}_{r}^{\ell}, \underline{q}_{r}^{\ell}-\underline{Q}_{r}^{\ell}\right)+C\left|\left(I-P^{h}\right) k\right|_{0, \infty, \Omega}\left|\underline{Q}_{r}^{\ell}\right|_{0, r, \Omega}^{r} .
\end{aligned}
$$

The desired result (3.7c), and hence (3.7d), for $n=\ell$ follow from combining (3.16)(3.18) and noting (2.2), (3.7a) for $n=\ell,(3.7 \mathrm{c})$ for $n=\ell-1$, the weak convergence versions of $(3.7 \mathrm{c}, \mathrm{d})$ for $n=\ell$ and $(2.11)$.

It follows from (3.10) and the above induction step that the desired results (3.7a-d) hold for the stated range of $n$; and furthermore, $\left\{\underline{q}_{r}^{n}, b_{r}^{n}\right\}_{n=1}^{N}$ is the unique solution of $\left(\mathrm{Q}_{r}^{\tau}\right)$. The first five bounds in (3.8) then follow directly from $(2.18 \mathrm{a}, \mathrm{b})$, $(3.7 \mathrm{a}-\mathrm{d})$ and $(2.5 \mathrm{a})$.

Finally, we need to prove the sixth bound in (3.8). First, we note from (3.1b), (A1) and the first bound in (3.8) that for $n=1 \rightarrow N$

$$
\begin{aligned}
\left|\left(F\left(b_{r}^{n}\right)-F\left(b_{e}^{n}\right), \underline{\nabla} \cdot \underline{v}\right)\right| & \leq k_{1}\left|\underline{q}_{r}^{n}\right|_{0, r, \Omega}^{r-1}|\underline{v}|_{0, r, \Omega} \leq\left[\frac{r-1}{r}\left|\underline{q}_{r}^{n}\right|_{0, r, \Omega}^{r}+C\right]|\underline{v}|_{0, r, \Omega} \\
& \leq C(T)|\underline{v}|_{0, r, \Omega} \quad \forall \underline{v} \in \underline{V}^{r}(\Omega) .
\end{aligned}
$$

It follows from (3.19) for any integer $n \in[1, N]$, as $C_{0}^{\infty}(\Omega)$ is dense in $L^{r}(\Omega)$, that the distributional gradient of $F\left(b_{r}^{n}\right)$ belongs to the dual of $\left[L^{r}(\Omega)\right]^{2}$. Hence for $n=1 \rightarrow N$ we have that $\underline{\nabla}\left[F\left(b_{r}^{n}\right)\right] \in\left[L^{p}(\Omega)\right]^{2}$ and

$$
\left|\left(\underline{\nabla}\left[F\left(b_{r}^{n}\right)\right], \underline{v}\right)\right| \leq C(T)|\underline{v}|_{0, r, \Omega} \quad \forall \underline{v} \in\left[L^{r}(\Omega)\right]^{2} .
$$

Choosing $\underline{v}(\underline{x})=\left|\underline{\nabla}\left[F\left(b_{r}^{n}(\underline{x})\right)\right]\right|^{p-2} \underline{\nabla}\left[F\left(b_{r}^{n}(\underline{x})\right)\right]$ if $\left|\underline{\nabla}\left[F\left(b_{r}^{n}(\underline{x})\right)\right]\right| \neq 0$, and $\underline{v}(\underline{x})=\underline{0}$ otherwise, in the above and as $F$ is globally Lipschitz, recall (2.2) and (A1), we obtain the final bound in (3.8).

Let

$$
\begin{aligned}
\underline{q}_{r}^{+}(\cdot, t) & :=\underline{q}_{r}^{n}(\cdot), \quad b_{e}^{+}(t):=b_{e}^{n}, \quad b_{r}^{+}(\cdot, t):=b_{r}^{n}(\cdot), \\
b_{r}^{c}(\cdot, t) & :=\frac{\left(t-t_{n-1}\right)}{\tau_{n}} b_{r}^{n}(\cdot)+\frac{\left(t_{n}-t\right)}{\tau_{n}} b_{r}^{n-1}(\cdot) \quad t \in\left(t_{n-1}, t_{n}\right], \quad n \geq 1 .
\end{aligned}
$$


It follows from $(3.21),(3.8),(2.12)$ and as $b_{e} \in H^{1}(0, T)$ that

$$
\begin{array}{ccc}
b_{r}^{+}-b_{r}^{c} \rightarrow 0 \quad \text { strongly in } L^{2}\left(\Omega_{T}\right), & b_{e}^{+} \rightarrow b_{e} & \text { strongly in } L^{\infty}(0, T) \\
& \text { as } \tau \rightarrow 0 .
\end{array}
$$

Adopting the notation $(3.21),\left(\mathrm{Q}_{r}^{\tau}\right)$ can be restated as:

$$
\begin{aligned}
\int_{0}^{T}\left(\frac{\partial b_{r}^{c}}{\partial t}-\underline{\nabla} \cdot \underline{q}_{r}^{+}, \eta\right) \mathrm{d} t=0 & \forall \eta \in L^{2}\left(\Omega_{T}\right), \text { (3.23a) } \\
\int_{0}^{T}\left[\left(k\left|\underline{q}_{r}^{+}\right|^{r-2} \underline{q}_{r}^{+}, \underline{v}\right)+\left(F\left(b_{r}^{+}\right)-F\left(b_{e}^{+}\right), \underline{\nabla} \cdot \underline{v}\right)\right] \mathrm{d} t=0 & \forall \underline{v} \in L^{2}\left(0, T ; \underline{V}^{r}(\Omega)\right) .
\end{aligned}
$$

Theorem 3.2. Let the Assumptions (A1) and (A2) hold. For all time partitions $\left\{\tau_{n}\right\}_{n=1}^{N}$ and for all $r \in(1,2]$ such that $r \rightarrow 1$ as $\tau \rightarrow 0$; the unique solution $\left\{\underline{q}_{r}^{+}, b_{r}^{+}\right\}$ to $\left(Q_{r}^{\tau}\right)$ is such that there exists a subsequence $\left\{\underline{q}_{r}^{+}, b_{r}^{+}\right\}_{\tau_{j}>0}$ of $\left\{\underline{q}_{r}^{+}, b_{r}^{+}\right\}_{\tau>0}$ and $\{\underline{q}, b\} \in L^{2}\left(0, T ; \underline{V}^{\mathcal{M}}(\Omega)\right) \times\left[H^{1}\left(0, T ; L^{2}(\Omega)\right) \cap L^{2}\left(0, T ; H^{1}(\Omega)\right)\right]$ such that as $\tau_{j} \rightarrow 0$

$$
\begin{aligned}
\underline{q}_{r}^{+} & \rightarrow \underline{q} & & \text { vaguely in } L^{2}\left(0, T ;[\mathcal{M}(\bar{\Omega})]^{2}\right), \\
\underline{\nabla} \cdot \underline{q}_{r}^{+} & \rightarrow \underline{\nabla} \cdot \underline{q} & & \text { weakly in } L^{2}\left(\Omega_{T}\right), \\
b_{r}^{+}, b_{r}^{c} & \rightarrow b & & \text { weakly in } L^{2}\left(0, T ; H^{1}(\Omega)\right), \\
\frac{\partial b_{r}^{c}}{\partial t} & \rightarrow \frac{\partial b}{\partial t} & & \text { weakly in } L^{2}\left(\Omega_{T}\right), \\
b_{r}^{+}, b_{r}^{c} & \rightarrow b & & \text { strongly in } L^{2}\left(\Omega_{T}\right) .
\end{aligned}
$$

Moreover, $\{\underline{q}, b\}$ solves $(Q),(1.15 a, b)$ with $\Omega^{*} \equiv \Omega$.

Proof. The bound (3.8) yields immediately, on noting that $r \in(1,2],(3.21)$ and our assumption (A1) on $k$, the first four bounds in

$$
\int_{0}^{T}\left[\left|\underline{\nabla} \cdot \underline{q}_{r}^{+}\right|_{0, \Omega}^{2}+\left\|b_{r}^{+}\right\|_{1, \Omega}^{2}+\left\|b_{r}^{c}\right\|_{1, \Omega}^{2}+\left|\frac{\partial b_{r}^{c}}{\partial t}\right|_{0, \Omega}^{2}+\left|\underline{q}_{r}^{+}\right|_{0, r, \Omega}^{2}\right] \mathrm{d} t \leq C(T) .
$$

Next we have from (3.1b), with $\underline{v} \equiv \underline{q}_{r}^{n}$, (A1) and (3.8) that for $n=1 \rightarrow N$

$$
k_{0}\left|\underline{q}_{r}^{n}\right|_{0, r, \Omega}^{r} \leq\left(F\left(b_{e}^{n}\right)-F\left(b_{r}^{n}\right), \underline{\nabla} \cdot \underline{q}_{r}^{n}\right) \leq C(T)\left|\underline{\nabla} \cdot \underline{q}_{r}^{n}\right|_{0, \Omega} .
$$

Therefore the last bound in (3.25) follows immediately from (3.26), (3.21) and the first bound in (3.25).

The subsequence convergence results $(3.24 \mathrm{a}-\mathrm{d})$ follow immediately from (3.25) and (3.22) The strong convergence result (3.24e) follows from $(3.24 \mathrm{c}, \mathrm{d})$, a standard compactness result and $(3.22)$. As $b_{r}^{c}(\cdot, 0)=b^{0}(\cdot)$, it follows from the above that $b(\cdot, 0)=b^{0}(\cdot)$.

It follows immediately from passing to the limit $\tau_{j} \rightarrow 0$ in (3.23a), on noting $(3.24 \mathrm{~b}, \mathrm{~d})$ and $(3.22)$, that $\{\underline{q}, b\}$ satisfy $(1.15 \mathrm{a})$ with $\Omega^{*} \equiv \Omega$. 
Given any $\underline{z} \in L^{2}\left(0, T ;\left[C^{\infty}(\bar{\Omega})\right]^{2}\right)$, we choose $\underline{v} \equiv \underline{q}_{r}^{+}-\underline{z}$ in (3.23b) to yield, on noting $(2.5 \mathrm{a})$, that

$$
\begin{aligned}
-\int_{0}^{T}\left(F\left(b_{r}^{+}\right)-F\left(b_{e}^{+}\right), \underline{\nabla} \cdot\left(\underline{q}_{r}^{+}-\underline{z}\right)\right) \mathrm{d} t & =\int_{0}^{T}\left(k\left|\underline{q}_{r}^{+}\right|^{r-2} \underline{q}_{r}^{+}, \underline{q}_{r}^{+}-\underline{z}\right) \mathrm{d} t \\
& \geq r^{-1} \int_{0}^{T}\left(k\left[\left|\underline{q}_{r}^{+}\right|^{r}-|\underline{z}|^{r}\right], 1\right) \mathrm{d} t .
\end{aligned}
$$

It follows immediately from $(3.24 \mathrm{~b}, \mathrm{e}),(2.2)$ and our assumptions on $M$ that for any $\underline{z} \in L^{2}\left(0, T ;\left[C^{\infty}(\bar{\Omega})\right]^{2}\right)$

$$
\int_{0}^{T}\left(F\left(b_{r}^{+}\right)-F\left(b_{e}^{+}\right), \underline{\nabla} \cdot\left(\underline{q}_{r}^{+}-\underline{z}\right)\right) \mathrm{d} t \rightarrow \int_{0}^{T}\left(F(b)-F\left(b_{e}\right), \underline{\nabla} \cdot(\underline{q}-\underline{z})\right) \mathrm{d} t
$$

Next, we note that for any $\underline{z} \in L^{2}\left(0, T ;\left[C^{\infty}(\bar{\Omega})\right]^{2}\right)$

$$
r^{-1} \int_{0}^{T}\left(k|\underline{z}|^{r}, 1\right) \mathrm{d} t \rightarrow \int_{0}^{T}(k|\underline{z}|, 1) \mathrm{d} t \quad \text { as } \quad \tau_{j} \rightarrow 0 .
$$

Finally, it follows from (3.24a), and similarly to (1.19), that

$$
\liminf _{\tau_{j} \rightarrow 0} r^{-1} \int_{0}^{T}\left(k\left|\underline{q}_{r}^{+}\right|^{r}, 1\right) \mathrm{d} t \geq \liminf _{\tau_{j} \rightarrow 0} \int_{0}^{T}\left(k\left|\underline{q}_{r}^{+}\right|, 1\right) \mathrm{d} t \geq \int_{0}^{T}\left[\int_{\bar{\Omega}} k|\underline{q}|^{r}\right] \mathrm{d} t .
$$

Combining equations (3.27)-(3.30), it follows that $\{q, b\}$ satisfy (1.15b) for any $\underline{v} \in L^{2}\left(0, T ;\left[C^{\infty}(\bar{\Omega})\right]^{2}\right)$. The desired result, $\{\underline{q}, b\}$ satisfies $(1.15 \mathrm{~b})$ with $\Omega^{*} \equiv \Omega$ for any $\underline{v} \in L^{2}\left(0, T ; \underline{V}^{\mathcal{M}}(\Omega)\right)$, and hence $\{\underline{q}, b\}$ solves $(\mathrm{Q})$ with $\Omega^{*} \equiv \Omega$, then follows from the density results $(1.22 \mathrm{a}-\mathrm{c})$.

Theorem 3.3. Let the assumptions of Theorem 3.2 hold. In addition, let $M \in C(\mathbb{R}$, $\left.\left[M_{0}, M_{1}\right]\right)$. We then have that any solution $\{\underline{q}, b\}$ of $(Q)$ with $\Omega^{*} \equiv \Omega$ is such that $w=b-b_{e} \in L^{2}\left(0, T ; K\left(w+b_{e}\right)\right) \cap H^{1}\left(0, T ; L^{2}(\Omega)\right)$ and $w(\cdot, 0)=w^{0}(0)$. In addition, we have that

$$
\int_{0}^{T}\left[\int_{\bar{\Omega}} k M\left(w+b_{e}\right)|\underline{q}|+(w, \underline{\nabla} \cdot \underline{q})\right] \mathrm{d} t=0 .
$$

Moreover, $w$ solves the quasi-variational inequality $(P),(1.8)$, with $\Omega^{*} \equiv \Omega$.

Proof. We adapt the proof of Theorem 3.2 in Barrett and Prigozhin ${ }^{6}$. Any solution $\{q, b\}$ of $(\mathrm{Q})$ yields that $w=b-b_{e} \in H^{1}\left(0, T ; L^{2}(\Omega)\right)$ and $w(\cdot, 0)=w^{0}(\cdot)$. Let

$$
Z(\underline{v}):=\int_{0}^{T}\left[\int_{\bar{\Omega}} k|\underline{v}|+\left(F(b)-F\left(b_{e}\right), \underline{\nabla} \cdot \underline{v}\right)\right] \mathrm{d} t .
$$

It follows from (1.15b) with $\Omega^{*} \equiv \Omega$ and (3.32) that

$$
Z(\underline{q})=\mathcal{Z}:=\min _{\underline{v} \in L^{2}\left(0, T ; \underline{V}^{\mathcal{M}}(\Omega)\right)} Z(\underline{v}) \leq Z(\underline{0})=0 .
$$


If $\mathcal{Z}<0$ then, for any minimizing sequence $\left\{\underline{v}_{j}\right\}$, we obtain that $Z\left(2 \underline{v}_{j}\right) \rightarrow 2 \mathcal{Z}<\mathcal{Z}$, which is a contradiction. Hence $\mathcal{Z}=0$, and so we have that $Z(\underline{v}) \geq 0=Z(\underline{q})$ for any $\underline{v} \in L^{2}\left(0, T ; \underline{V}^{\mathcal{M}}(\Omega)\right)$. Since this is true also for $-\underline{v}$, we obtain that

$$
\begin{aligned}
\left|\int_{0}^{T}\left(F\left(b_{e}\right)-F(b), \underline{\nabla} \cdot \underline{v}\right) \mathrm{d} t\right| \leq \int_{0}^{T}\left[\int_{\bar{\Omega}} k|\underline{v}|\right] \mathrm{d} t \\
\forall \underline{v} \in L^{2}\left(0, T ; \underline{V}^{\mathcal{M}}(\Omega)\right), \\
\text { and } \quad \int_{0}^{T}\left(F\left(b_{e}\right)-F(b), \underline{\nabla} \cdot \underline{q}\right) \mathrm{d} t=\int_{0}^{T}\left[\int_{\bar{\Omega}} k|\underline{q}|\right] \mathrm{d} t .
\end{aligned}
$$

It follows from (3.34a), as $C_{0}^{\infty}\left(0, T ;\left[C_{0}^{\infty}(\Omega)\right]^{2}\right)$ is dense in $L^{1}\left(0, T ;\left[L^{1}(\Omega)\right]^{2}\right)$ and $k \in C(\bar{\Omega})$, that the distributional gradient of $F(b)$ belongs to the dual of $L^{1}\left(0, T ;\left[L^{1}(\Omega)\right]^{2}\right)$. Hence, as $k(\underline{x}) \geq k_{0}>0$ for all $\underline{x} \in \bar{\Omega}$, we have that $\underline{\nabla}[F(b)] \in L^{\infty}\left(0, T ;\left[L^{\infty}(\Omega)\right]^{2}\right)$ and

$$
\left|\int_{0}^{T}\left(k^{-1} \underline{\nabla}[F(b)], \underline{v}\right) \mathrm{d} t\right| \leq\|\underline{v}\|_{L^{1}\left(\Omega_{T}\right)} \quad \forall \underline{v} \in L^{1}\left(0, T ;\left[L^{1}(\Omega)\right]^{2}\right) .
$$

For any $p \in[1, \infty)$, choosing $\underline{v}(\underline{x}, t)=\left|[k(\underline{x})]^{-1} \underline{\nabla}[F(b(\underline{x}, t))]\right|^{p-2}[k(\underline{x})]^{-1}$ $\underline{\nabla}[F(b(\underline{x}, t))]$ if $|\underline{\nabla}[F(b(\underline{x}, t))]| \neq 0$, and $\underline{v}(\underline{x}, t)=\underline{0}$ otherwise, in the above, and noting the continuity of the $p$ norm for $p \in[1, \infty]$, we obtain that

$$
\left\|k^{-1} \underline{\nabla}[F(b)]\right\|_{L^{\infty}\left(\Omega_{T}\right)} \leq 1 .
$$

It follows from (3.34a) and (3.36) that

$$
\left|\int_{0}^{T}\left(\int_{\partial \Omega}\left[F(b)-F\left(b_{e}\right)\right] \underline{v} \cdot \underline{n} \mathrm{~d} s\right) \mathrm{d} t\right| \leq 2 k_{1}\|\underline{v}\|_{L^{1}\left(\Omega_{T}\right)} \quad \forall \underline{v} \in L^{2}\left(0, T ; \underline{V}^{2}(\Omega)\right),
$$

where $\underline{n}$ is the outward unit normal to $\partial \Omega$. Without loss of generality, we can assume that $\Omega$ is strictly star-shaped with respect to the origin and so $[\underline{x} \cdot \underline{n}(\underline{x})] \geq n_{0}>0$ for a.e. $\underline{x} \in \partial \Omega$. Choosing $\underline{v}=\phi_{j}\left[F(b)-F\left(b_{e}\right)\right] \underline{x}$ in $(3.37)$, where $\phi_{j} \in H^{1}(\Omega)$ is such that $\phi_{j}=1$ on $\partial \Omega$ and $\left\|\phi_{j}\right\|_{L^{1}(\Omega)} \rightarrow 0$ as $j \rightarrow \infty$, yields that $F(b)=F\left(b_{e}\right)$ a.e. on $\partial \Omega \times(0, T)$ and hence $b=b_{e}$ a.e. on $\partial \Omega \times(0, T)$. For example, one can choose $\phi_{j}$ in the following way. For integers $j \geq 1$, let $\phi_{j} \in H^{1}(\Omega)$ be the extension by zero to $\Omega_{j}:=\frac{j-1}{j} \Omega$ of the unique solution of Laplace's equation in $\Omega \backslash \Omega_{j}$ satisfying the Dirichlet boundary conditions $\phi_{j}=1$ on $\partial \Omega$ and $\phi_{j}=0$ on $\partial \Omega_{j}$. The weak maximum principle yields that $\phi_{j}(\underline{x}) \in[0,1]$ for a.e. $\underline{x} \in \Omega$, and so $\left\|\phi_{j}\right\|_{L^{1}(\Omega)} \rightarrow 0$ as $j \rightarrow \infty$. Combining $b=b_{e}$ a.e. on $\partial \Omega \times(0, T)$ with (3.36) yields, on recalling (2.2), that $w=b-b_{e} \in L^{2}\left(0, T ; K\left(w+b_{e}\right)\right)$ with $\Omega^{*} \equiv \Omega$.

Let $\left\{\underline{q}_{j}\right\}_{j \geq 1} \in L^{2}\left(0, T ;\left[C^{\infty}(\bar{\Omega})\right]^{2}\right)$ satisfy $(1.22 \mathrm{a}-\mathrm{c})$, with $\underline{v} \equiv \underline{q}$, for a.a. $t \in$ 
$(0, T)$. It follows as $w \in L^{2}\left(0, T ; K\left(w+b_{e}\right)\right)$ that

$$
\begin{aligned}
0 & \leq \int_{\Omega_{T}}\left[k M(b)\left|\underline{q}_{j}\right|+w \underline{\nabla} \cdot \underline{q}_{j}\right] \mathrm{d} \underline{x} \mathrm{~d} t=\int_{\Omega_{T}}\left[k M(b)\left|\underline{q}_{j}\right|-\underline{\nabla} w \cdot \underline{q}_{j}\right] \mathrm{d} \underline{x} \mathrm{~d} t \\
& =\int_{\Omega_{T}} M(b)\left[k\left|\underline{q}_{j}\right|-\underline{\nabla}[F(b)] \cdot \underline{q}_{j}\right] \mathrm{d} \underline{x} \mathrm{~d} t \leq M_{1} \int_{\Omega_{T}}\left[k\left|\underline{q}_{j}\right|-\underline{\nabla}[F(b)] \cdot \underline{q}_{j}\right] \mathrm{d} \underline{x} \mathrm{~d} t \\
& =M_{1} \int_{\Omega_{T}}\left[k\left|\underline{q}_{j}\right|+\left[F(b)-F\left(b_{e}\right)\right] \underline{\nabla} \cdot \underline{q}_{j}\right] \mathrm{d} \underline{x} \mathrm{~d} t .
\end{aligned}
$$

Passing to the limit $j \rightarrow \infty$ in (3.38), on noting (1.22a-c), (3.34b) and that $M \in$ $C\left(\mathbb{R},\left[M_{0}, M_{1}\right]\right)$, yields the desired result (3.31). Similarly, we have for any $\xi \in$ $K\left(w+b_{e}\right)$ that

$$
\int_{\Omega_{T}} \xi \underline{\nabla} \cdot \underline{q}_{j} \mathrm{~d} \underline{x} \mathrm{~d} t=-\int_{\Omega_{T}} \underline{\nabla} \xi \cdot \underline{q}_{j} \mathrm{~d} \underline{\mathrm{x}} \mathrm{d} t \geq-\int_{\Omega_{T}} k M\left(w+b_{e}\right)\left|\underline{q}_{j}\right| \mathrm{d} \underline{x} \mathrm{~d} t ;
$$

and hence that

$$
\int_{0}^{T}(\xi, \underline{\nabla} \cdot \underline{q}) \mathrm{d} t \geq-\int_{0}^{T}\left[\int_{\bar{\Omega}} k M\left(w+b_{e}\right)|\underline{q}|\right] \mathrm{d} t .
$$

Choosing $\eta \equiv \xi-w$ in (1.15a) with $\Omega^{*} \equiv \Omega$ and $\xi \in L^{2}\left(0, T ; K\left(w+b_{e}\right)\right)$, and noting (3.31) and (3.40), yields that $w$ solves the primal variational inequality $(\mathrm{P})$, (1.8).

\section{Numerical Experiments}

To compute the unique solution $\left\{\underline{Q}_{r}^{n}, B_{r}^{n}\right\}$ of our approximation $\left(Q_{r}^{h, \tau}\right)$ at the $\mathrm{n}^{\text {th }}$ time step we substitute (2.19) into (2.13b). This yields a nonlinear problem for $\underline{Q}:=\underline{Q}_{r}^{n} \in \underline{V}^{h}:$

$$
\left(k|\underline{Q}|^{r-2} \underline{Q}, \underline{v}^{h}\right)^{h}+\left(F\left(B_{r}^{n-1}+\tau_{n} \underline{\nabla} \cdot \underline{Q}\right)-F\left(b_{e}^{n}\right), \underline{\nabla} \cdot \underline{v}^{h}\right)=0 \quad \forall \underline{v}^{h} \in \underline{V}^{h},
$$

which we solved iteratively. At the $(j+1)^{t h}$ iteration, we first solve the following linear problem for $\underline{Q}^{j+\frac{1}{2}} \in \underline{V}^{h}$ :

$$
\begin{aligned}
& \left(F\left(B_{r}^{n-1}+\tau_{n} \underline{\nabla} \cdot \underline{Q}^{j}\right), \underline{\nabla} \cdot \underline{v}^{h}\right) \\
& \quad+\tau_{n}\left(\left[M\left(B_{r}^{n-1}+\tau_{n} \underline{\nabla} \cdot \underline{Q}^{j}\right)\right]^{-1} \underline{\nabla} \cdot\left(\underline{Q}^{j+\frac{1}{2}}-\underline{Q}^{j}\right)-F\left(b_{e}^{n}\right), \underline{\nabla} \cdot \underline{v}^{h}\right) \\
& \quad+\left(k\left[\left|\underline{Q}^{j}\right|^{r-2} \underline{Q}^{j}+\left|\underline{Q}^{j}\right|_{\varepsilon}^{r-2}\left(\underline{Q}^{j+\frac{1}{2}}-\underline{Q}^{j}\right)\right], \underline{v}^{h}\right)^{h}=0 \quad \forall \underline{v}^{h} \in \underline{V}^{h},
\end{aligned}
$$

where $|\underline{v}|_{\varepsilon}:=\left(|\underline{v}|^{2}+\varepsilon^{2}\right)^{\frac{1}{2}}$ with $\varepsilon \ll 1$, and we have recalled that $F^{\prime}(s)=[M(s)]^{-1}$. Clearly, the linear system (4.2) is well-posed. Finally we set $\underline{Q}^{j+1}=\alpha \underline{Q}^{j+\frac{1}{2}}+(1-$ a) $Q^{j}$, where $\alpha \geq 1$ is an over-relaxation parameter. In all of our examples below, we chose $r=1+10^{-7}, \varepsilon=10^{-9}$ and $\alpha=1.2$.

Let $\mathcal{E}^{h}$ be the set of edges of $\mathcal{T}^{h}$, and $\underline{\phi}_{e}(\underline{x})$ be the vector basis function associated with the edge $e \in \mathcal{E}^{h}$ in the Raviart-Thomas finite element space $\underline{V}^{h}$, see e.g. Bahriawati and Carstensen ${ }^{4}$ for details. Then any $\underline{v}^{h} \in \underline{V}^{h}$ can be represented as 
$\sum_{e \in \mathcal{E}^{h}} v_{e}^{h} \underline{\phi}_{e}$, and we define $\left\|\underline{v}^{h}\right\|_{\mathcal{E}^{h}}:=\sum_{e \in \mathcal{E}^{h}}\left|v_{e}^{h}\right||e|$. Our stopping criterion for the above iterative scheme was

$$
\frac{\left\|\underline{Q}^{j+1}-\underline{Q}^{j}\right\|_{\mathcal{E}^{h}}}{\left\|\underline{Q}^{j+1}\right\|_{\mathcal{E}^{h}}} \leq \varepsilon_{N L}
$$

where $\varepsilon_{N L}$ is a given tolerance. We set $\varepsilon_{N L}=2 \cdot 10^{-4}$ throughout the examples below. When this stopping criterion was satisfied, we set $\underline{Q}_{r}^{n}=\underline{Q}^{j+1}$ and computed the magnetic field $B_{r}^{n}$ using (2.19). We used the Matlab PDE Toolbox for the domain triangulation, and curved domains were approximated by polygons. The finite element meshes in our examples below contained about seven thousand triangles.

As in Barrett and Prigozhin ${ }^{6}$, the parameters in the numerical simulations were chosen on assuming that the dimensionless variables $(\underline{x}, t, \cdots)$ were obtained from the original variables $\left(\underline{x}^{\prime}, t^{\prime}, \cdots\right)$ as follows:

$$
\underline{x}=\frac{\underline{x}^{\prime}}{L}, \quad t=\frac{t^{\prime}}{t_{0}}, \quad \underline{j}=\frac{j^{\prime}}{j_{0}}, \quad b=\frac{b^{\prime}}{L j_{0}}, \quad \underline{e}=\frac{\underline{e}^{\prime} t_{0}}{L^{2} j_{0}},
$$

where $L$ is the characteristic cross section size (the maximal horizontal extension in the plots below), $j_{0}$ is the value of the critical current density $J_{c}$ for a zero magnetic field, and the superconductors were homogeneous with $k(\underline{x}) \equiv 1$. In the examples below we assumed that $\left|d_{t} b_{e}^{\prime}\right|$ is a constant, which was scaled to unity by choosing the time scale $t_{0}$ appropriately.

In our numerical simulations, the time step was uniform with $\tau=0.005$. Initially, the magnetic field was zero, i.e. $w^{0} \equiv b_{e}(0)=0$, and we assumed a growing external field, $b_{e}(t)=t$; except for the last example on hysteresis.

As our first example we compare, see Fig. 1, for a rectangular cross section $\Omega$, the Bean model $\left(J_{c}=1\right.$ in dimensionless variables $)$ with the Kim model, $J_{c}(s)=\left(1+\frac{|s|}{a}\right)^{-1}$; here and below we set $a=0.02$ for this model. Since the critical current density in the Kim model decreases with the growth of magnetic field, the shielding eddy current is weaker and magnetic field penetrates further inside the superconductor; this field, $b=\widetilde{b}$, is given by (1.10). To estimate the accuracy of our numerical solution $B_{r}^{n}$ we compared it with $\widetilde{B}^{n} \in S^{h}$, where $\left.\widetilde{B}^{n}\right|_{\sigma}=\widetilde{b}\left(\underline{x}_{\sigma}, t_{n}\right)$ and $\underline{x}_{\sigma}$ is the centroid of $\sigma$ for all $\sigma \in \mathcal{T}^{h}$. We obtained that

$$
\frac{\left\|\widetilde{B}^{n}-B_{r}^{n}\right\|_{0,1, \Omega}}{\left\|\widetilde{B}^{n}-b_{e}\left(t_{n}\right)\right\|_{0,1, \Omega}}<0.002 .
$$

The electric field found using Kim's model is stronger, but qualitatively similar to that in the Bean mode ${ }^{10}$. It has the same zig-zag shape, is zero in the zero-magneticfield core, it is parallel to level contours of the magnetic field, and vanishes along the discontinuity lines of the current density. Near concave corners of $\Omega$ the electric field becomes singular, see Fig. 2 where $\Omega$ is a circle with a section removed.

Although our analysis in Sections 2 and 3 holds only for continuous $k(\underline{x}) \geq$ $k_{0}>0$, our numerical method $\left(\mathrm{Q}_{r}^{h, \tau}\right)$ extends to piecewise constant $k$, where the discontinuities are aligned with the mesh. Therefore one can simulate numerically 

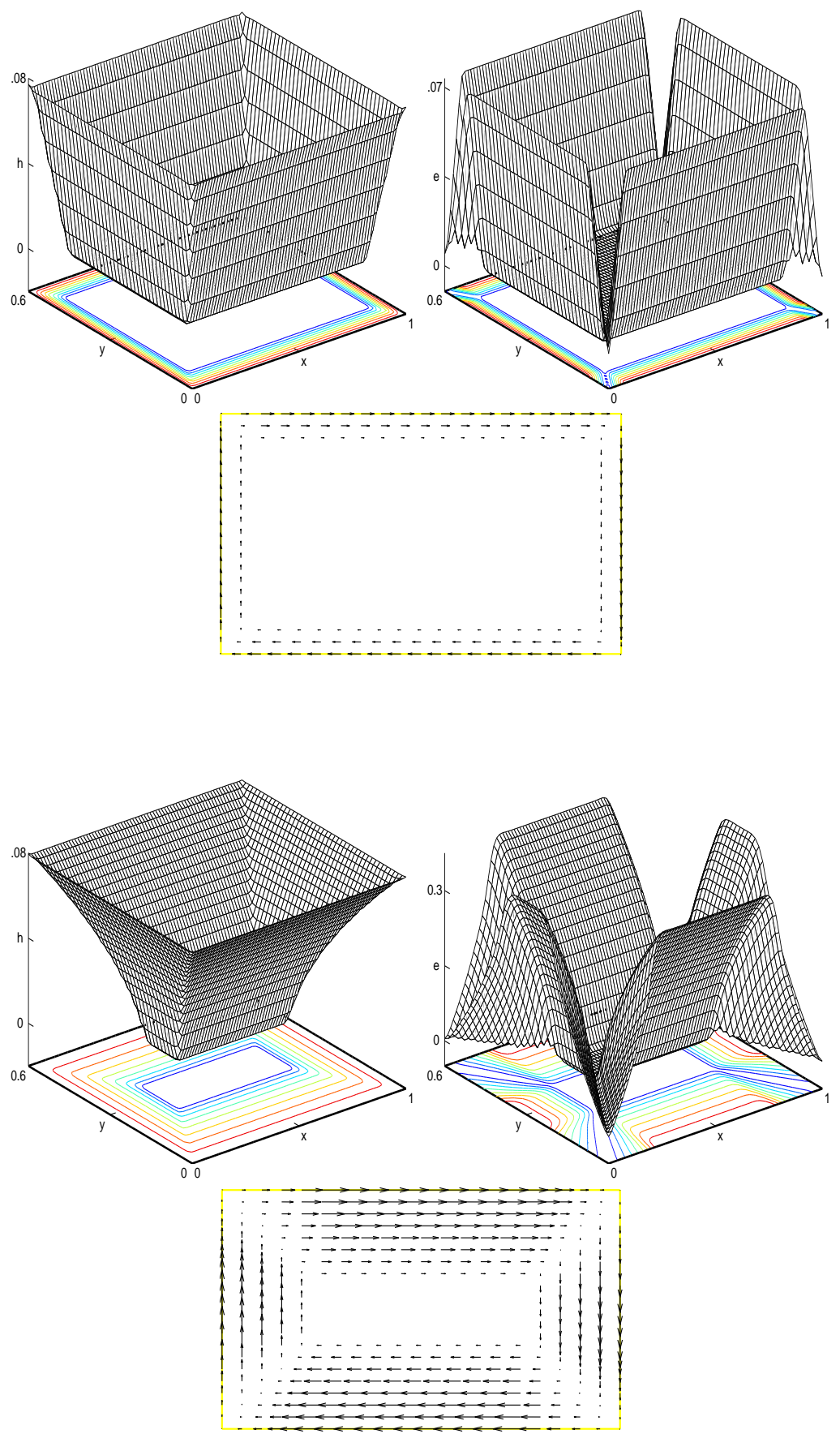

Fig. 1. Rectangular cross section, $b_{e}=t=0.08$. The Bean model (top) and the Kim model (bottom). The magnetic field (left) and the electric field (middle - $|\underline{e}|$, right - the vector field). 
the magnetisation of a superconductor with a multiply connected cross section, see Fig. 3, by filling the hole and setting $k=k_{0} \ll 1$ there. For this example, we chose $k_{0}=10^{-6}$ and so the eddy current in the hole is negligible; as in the other examples, $k \equiv 1$ in the superconductor. Similarly to the Bean model ${ }^{6}$ when the penetration zone reaches the hole boundary the magnetic field begins to penetrate the hole via an infinitesimally thin channel and the electric field becomes singular. This singularity is evident in Fig. 3, although the channel is slightly smeared by the relatively coarse mesh. For a cross section with only one hole, $\Omega_{1}$, we have that $\operatorname{dist}_{D}\left(\underline{x}, \partial \Omega^{*}\right)=\min \left\{\operatorname{dist}\left(\underline{x}, \partial \Omega^{*}\right), \operatorname{dist}\left(\underline{x}, \partial \Omega_{1}\right)+\operatorname{dist}\left(\partial \Omega_{1}, \partial \Omega^{*}\right)\right\}$. Calculating this distance is not difficult and we can substitute it into the derived analytical solution for the magnetic field, see Subsection 1.2, for multiply connected cross sections. The relative error in the $L^{1}$ norm, estimated as in the first example, was 0.011 for a mesh with about seven thousand elements and 0.007 for a refined mesh with about fifteen thousand elements (the same time step, $\tau=0.005$, was used in both cases).

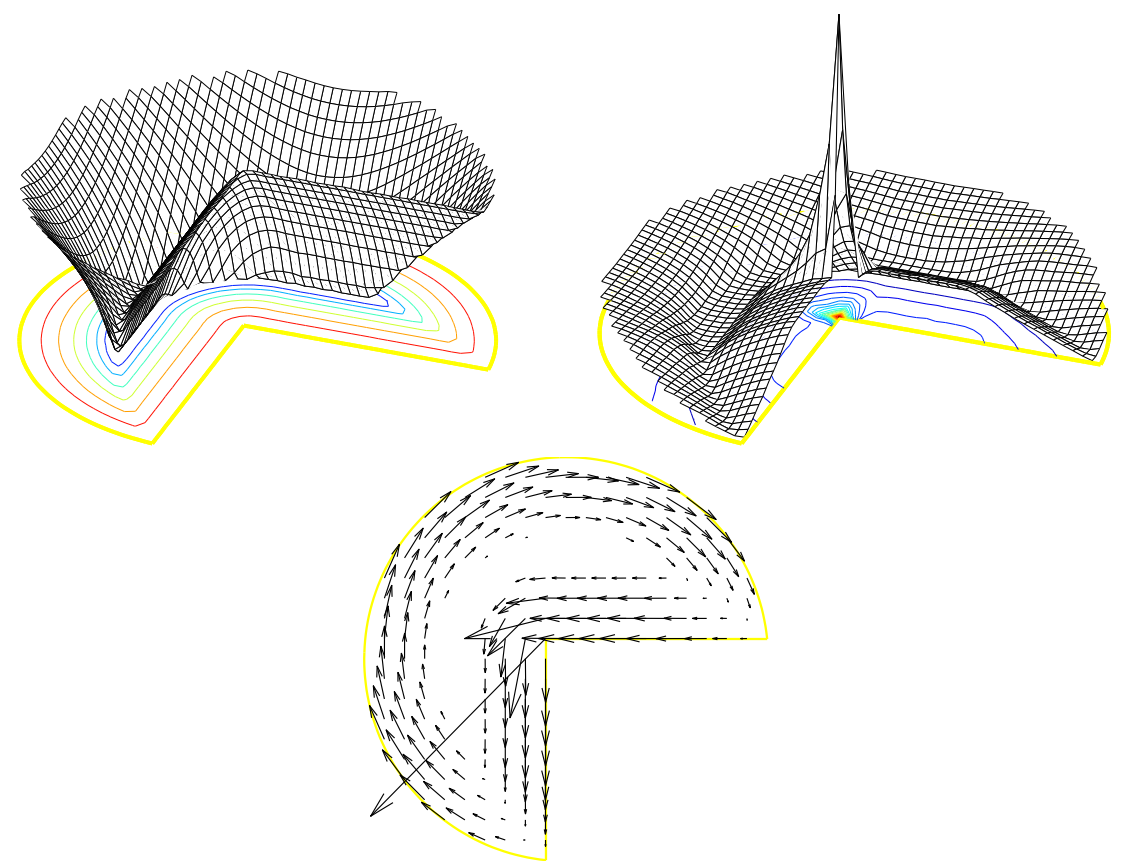

Fig. 2. Circular cross section with a section removed, $b_{e}=t=0.07$, and the Kim model. The magnetic field (left) and the electric field (middle - $|\underline{e}|$, right - the vector field). 
The difference between various critical state models is best exhibited by the corresponding magnetisation loops, showing the behavior of the magnetic momentum of a superconductor when the external field changes cyclically. For the longitudinal configuration considered in this paper, the magnetic moment of a superconductor per unit of length is $m=\int_{\Omega}\left(b(\underline{x}, t)-b_{e}(t)\right) \mathrm{d} \underline{x}$. The hysteresis loops in Fig. 4 were computed for the superconductor with a rectangular cross section as in the first example for three different models: the Bean model, the Kim model, and a model with a secondary peak in the $J_{c}(\cdot)$ dependence. In the latter case the critical current density was taken similar to that in Johansen et al. ${ }^{19}$; that is, $J_{c}(s)=\left(1+\frac{|s|}{a} \mid\right)^{-1}+c_{1}\left(\left(\frac{|s|}{a}-c_{2}\right)^{2}+c_{3}^{2}\right)^{-1}$, in dimensionless variables, with $a=0.02$ as in the Kim model, $c_{1}=1, c_{2}=8$ and $c_{3}=1$.

In conclusion, we note that although quasi-variational inequalities, arising in critical-state problems with critical current density depending on the magnetic field, are much more difficult mathematical problems than the variational inequalities arising in Bean's model, their numerical solution based on the dual formulation presented in this work is practically as efficient as the solution of the Bean model problems in Barrett and Prigozhin ${ }^{6}$.

\section{Acknowledgement}

We acknowledge support of the Sixth EU Framework Programme - Transnational Access implemented as Specific Support Action (Dryland Research SSA).

\section{References}

1. A. Azevedo and L. Santos, Convergence of convex sets with gradient constraint, $J$. Convex Analysis 11 (2004) 285-301.

2. A. Badía and C. López, The critical state in type II superconductors with cross-flow effects, J. Low Temp. Phys. 130 (2003) 129-153.

3. A. Badía-Majós and C. López, Electric field in hard superconductors with arbitrary cross section and general critical current law, J. Appl. Phys. 95 (2004) 8035-8040.

4. C. Bahriawati and C. Carstensen, Three Matlab implementations of the lowestorder Raviart-Thomas MFEM with a posteriori error control, Comput. Methods Appl. Math. 5 (2005) 333-361.

5. J.W. Barrett and L. Prigozhin, Bean's critical-state model as the $p \rightarrow \infty$ limit of an evolutionary $p$-Laplacian, Nonlinear Analysis 42 (2000) 977-993.

6. J. W. Barrett and L. Prigozhin, Dual formulations in critical state problems, Interfaces and Free Boundaries 8 (2006) 347-368.

7. J. W. Barrett and L. Prigozhin, A mixed formulation of the Monge-Kantorovich equations, $M^{2} A N 41$ (2007) 1041-1060.

8. C. P. Bean, Magnetization of high-field superconductors, Rev. Mod. Phys. 36 (1964) 31-39.

9. A. Bossavit, Numerical modeling of superconductors in three dimensions - A model and a finite-element method, IEEE Trans. Magn. 30 (1994) 3363-3366.

10. E. H. Brandt, Electric field in superconductors with rectangular cross section, Phys. Rev. B 52 (1995) 15442-15457. 
11. G. Buttazzo and E. Stepanov, Transport density in Monge-Kantorovich problems with Dirichlet conditions, Discrete Cont. Dyn. S. 12 (2005) 607-628.

12. P. Cannarsa and P. Cardaliaguet, Representation of equilibrium solutions to the table problem for growing sandpiles, J. Eur. Math. Soc. 6 435-464 (2004).

13. S.-S. Chow, Finite element error estimates for non-linear elliptic equations of monotone type, Numer. Math. 54 (1989) 373-393.

14. G. Crasta and A. Malusa, A variational approach to the macroscopic electrodynamics of anisotropic hard superconductors, Arch. Rational Mech. Anal. 192 (2009) 87-115.

15. M. Farhloul, A mixed finite element method for a nonlinear Dirichlet problem, IMA J. Numer. Anal. 18 (1998) 121-132.

16. G. B. Folland, Real Analysis: Modern Techniques and their Applications (Second Edition) (Wiley-Interscience, New York, 1984).

17. U. Janfalk, Behaviour in the limit, as $p \rightarrow \infty$, of minimizers of functionals involving $p$-Dirichlet integrals, SIAM J. Math. Anal. 27 (1996) 341-360.

18. U. Janfalk, On a minimization problem for vector fields in $L^{1}$, Bull. London Math. Soc. 28 (1996) 165-176.

19. T. H. Johansen, M.R. Koblischka, H. Bratsberg, and P. O. Hetland, Critical-state model with a secondary high-field peak in $J_{c}(B)$, Phys. Rev. B 56 (1997) 11273-



Fig. 3. Cross section with a hole, $b_{e}=t=0.09$, and the Kim model. The magnetic field (left) and the electric field (level contours of $|\underline{e}|$ and vector field, right). 
11278.

20. Y. Kashima, On the double critical-state model for type-II superconductivity in 3D, $M^{2} A N 42$ (2008) 333-374.

21. Y.B. Kim, C.F. Hempstead and A.R. Strnad, Critical persistent currents in hard superconductors, Phys. Rev. Lett. 9 (1962) 306-309.

22. L. Prigozhin, On the Bean critical-state problem in superconductivity, Euro. J. of Applied Math. 7 (1996) 237-247.

23. J.F. Rodrigues and L. Santos, A parabolic quasi-variational inequlity arising in a superconductivity model, Ann. Scuola Norm. Sup. Pisa Cl. Sci. XXX1X (2000) 153169.

24. G. Strang, $L^{1}$ and $L^{\infty}$ approximation of vector fields in the plane, Lect. Notes in Num. Appl. Anal. 5 (1982) 273-288.

25. R. Temam, Mathematical Methods in Plasticity. (Gauthier-Villars, Paris, 1985).


Fig. 4. Hysteresis loops (dimensionless variables): Bean's model (left), Kim's model (middle), and the model with a secondary peak in $J_{c}(b)$ (right). 